\title{
Control of Hopf Bifurcation in Autonomous System Based on Washout Filter
}

\author{
Wenju Du, Yandong Chu, Jiangang Zhang, Yingxiang Chang, Jianning Yu, and Xinlei An \\ Department of Mathematics, Lanzhou Jiaotong University, Lanzhou, Gansu 730070, China \\ Correspondence should be addressed to Jiangang Zhang; zhangjg114502@gmail.com
}

Received 4 July 2013; Accepted 7 September 2013

Academic Editor: Erik Van Vleck

Copyright (C) 2013 Wenju Du et al. This is an open access article distributed under the Creative Commons Attribution License, which permits unrestricted use, distribution, and reproduction in any medium, provided the original work is properly cited.

In order to further understand a Lorenz-like system, we study the stability of the equilibrium points and the existence of Hopf bifurcation by center manifold theorem and normal form theory. More precisely, we designed a washout controller such that the equilibrium $E_{0}$ undergoes a controllable Hopf bifurcation, and by adjusting the controller parameters, we delayed Hopf bifurcation phenomenon of the equilibrium $E_{+}$. Besides, numerical simulation is given to illustrate the theoretical analysis. Finally, two possible electronic circuits are given to realize the uncontrolled and the controlled systems.

\section{Introduction}

Over the past decades, as we have seen, researchers have paid a great attention to the control of nonlinear dynamical systems exhibiting Hopf bifurcation phenomena, because the presence of bifurcation is very important in many physical, biological, and chemical nonlinear systems [1-3]. Chen et al. $[4,5]$ created a certain bifurcation at a desired location with preferred properties by appropriate control. And they developed a washout-filter-aided dynamic feedback control laws for the creation of Hopf bifurcations. Wei and Yang [6] proposed nonlinear control scheme to the system and the controlled system can exhibit codimensions one, two, and three Hopf bifurcations in a much larger parameter regain. An et al. [7] based on washout filter designed a state feedback controller for Hopf bifurcation of the nonlinear systems. Ma et al. [8] designed a bifurcation controller using the method of washout filter so as to control the dynamic bifurcation in power system. Sotomayor et al. [9] use the projection method described in [10] to calculate the first and second Lyapunov coefficients associated with Hopf bifurcations of the Watt governor system, and it was extended to the calculation of the third and fourth Lyapunov coefficients.

Recently, Dias et al. [11] studied the existence of singularly degenerate heteroclinic cycles for a suitable choice of the parameters in the following Lorenz-like system:

$$
\dot{x}=a(y-x), \quad \dot{y}=a(b x-x z), \quad \dot{z}=-c z+x y,
$$

where $(x, y, z) \in \mathbf{R}^{3}$ are the state variables and $(a, b, c) \in \mathbf{R}^{3}$ are real constants. The system (1) has a chaotic attractor when the real constants $a=1, b=1$, and $c=0.08$, as shown in Figures 1(a)-1(d).

Moreover, the dynamics of the system (1) can be characterized with its Lyapunov exponents which are computed numerically by Wolf algorithm proposed in [12], where the Lyapunov exponents $\lambda_{1}=0.0295, \lambda_{2}=0$, and $\lambda_{3}=-1.1092$ as shown in Figure 2(d) and the Lyapunov dimension $D_{K Y}=$ 2.02664. The time history, frequency spectrum, and Poincaré map in $x-y\{z=1.3\}$ plane of the chaotic attractor are shown in Figures 2(a), 2(b), and 2(c), respectively.

The system possesses three equilibria $E_{0}=(0,0,0)$ and $E_{ \pm}=( \pm \sqrt{b c}, \pm \sqrt{b c}, b)$ if $b c>0$. However, the Hopf bifurcation at the equilibrium $E_{0}$ does not occur. Dias et al. [11] studied the existence of singularly degenerated heteroclinic cycles for a suitable choice of the parameters at the equilibrium $E_{+}$. The system (1) has a transversal Hopf point at $E_{+}$for $c=a(b-1) /(b+1)>0, a>0, b>0$. And the equilibrium $E_{+}$is locally asymptotically stable in the parameter space $(a, b, c) \in$ $v, v=\{(a, b, c): a>0, b>0, c>a(b-1) /(b+1)\}$.

In this work, we will design a control laws such that our feedback system undergoes a controllable Hopf bifurcation. 


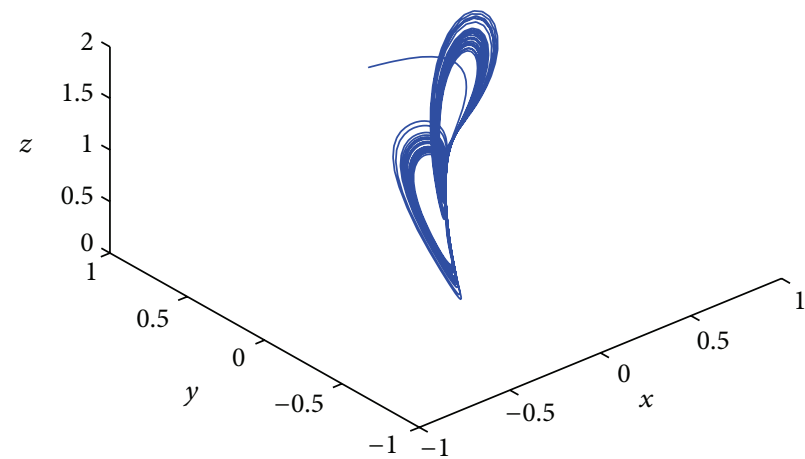

(a)

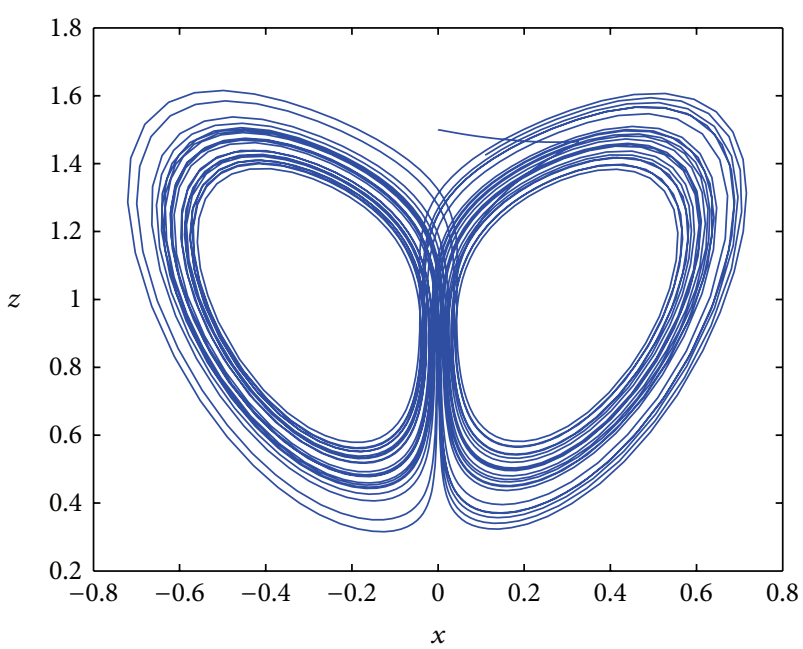

(c)

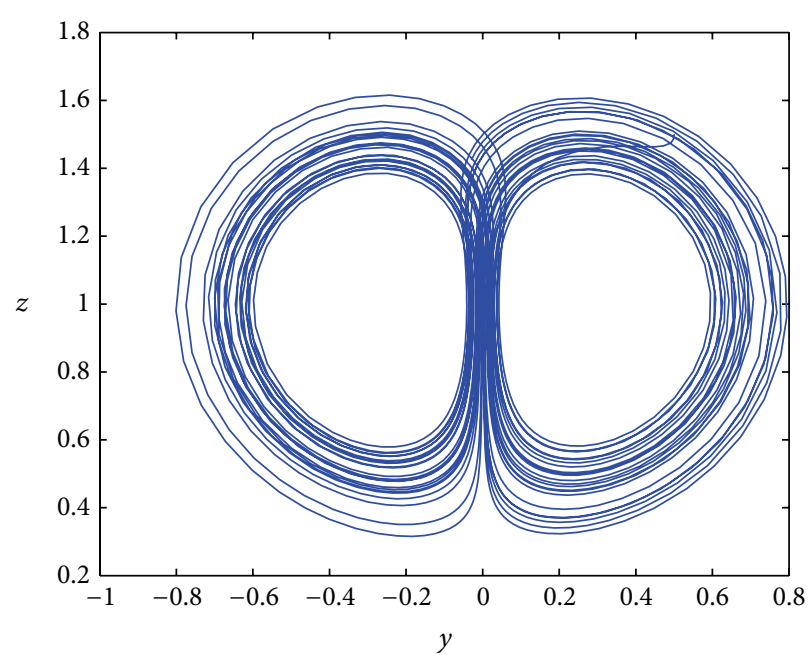

(b)

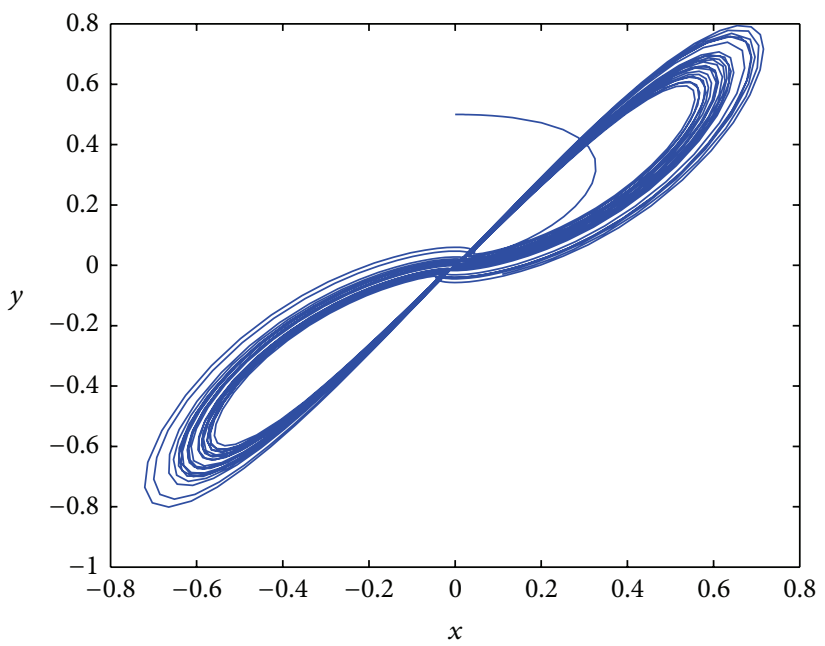

(d)

FIGURE 1: Phase trajectory in 3-D space and various projections of the chaotic attractor.

To accomplish the control of Hopf bifurcation in the system (1), we design the controller in the following structure:

$$
u=k_{1}(y-m v)+k_{2}(y-m v)^{3},
$$

where $k_{1}, k_{2}$ are the control gain vectors and $m$ is the washoutfilter time constant, which satisfied $m>0$ and $u(0)=0$.

This paper is organized as follows. In Section 2, a brief review of the methods used to study codimensions one and two Hopf bifurcations is presented. Through a linear analysis of system (16), we obtain the Hopf conditions for $E_{0}, E_{+}$, and the main results of this paper in Sections 3 and 4, respectively. In Section 5, two possible electronic circuits are given to realize the uncontrolled system and the controlled system. Finally, in Section 6 conclusion is given.

\section{Outline of the Hopf Bifurcation Methods}

This section is a review of the projection method described in $[9-11,13]$ for the calculation of the first Lyapunov coefficient and second Lyapunov coefficient associated with Hopf bifurcation, denoted by $l_{1}$ and $l_{2}$, respectively.

Consider the differential equation

$$
x^{\prime}=f(x, \zeta),
$$

where $x \in \mathbf{R}^{4}, \zeta \in \mathbf{R}^{m}$ are, respectively, vectors representing phase variables and control parameters. Assume that $f$ is of class $\mathbf{C}^{\infty}$ in $\mathbf{R}^{4} \times \mathbf{R}^{m}$. Suppose that (3) has an equilibrium point $x=x_{0}$ at $\zeta=\zeta_{0}$, and denoting the variable $x-x_{0}$ also by $x$, write

$$
F(x)=f\left(x, \zeta_{0}\right),
$$

as

$$
\begin{aligned}
F(x)= & A x+\frac{1}{2} B(x, x)+\frac{1}{6} C(x, x, x) \\
& +\frac{1}{24} D(x, x, x, x)+\frac{1}{120} E(x, x, x, x, x) \\
& +O\left(\|x\|^{6}\right),
\end{aligned}
$$




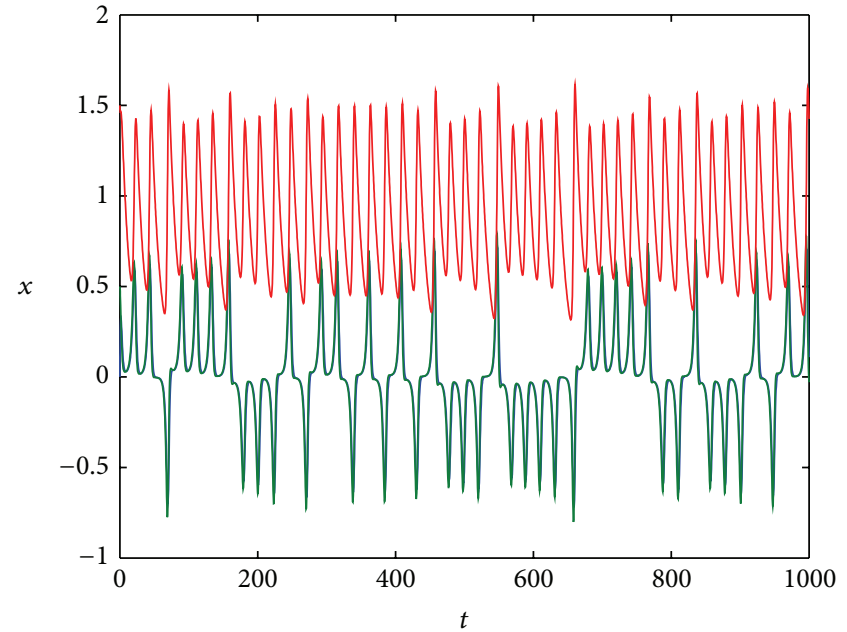

(a)

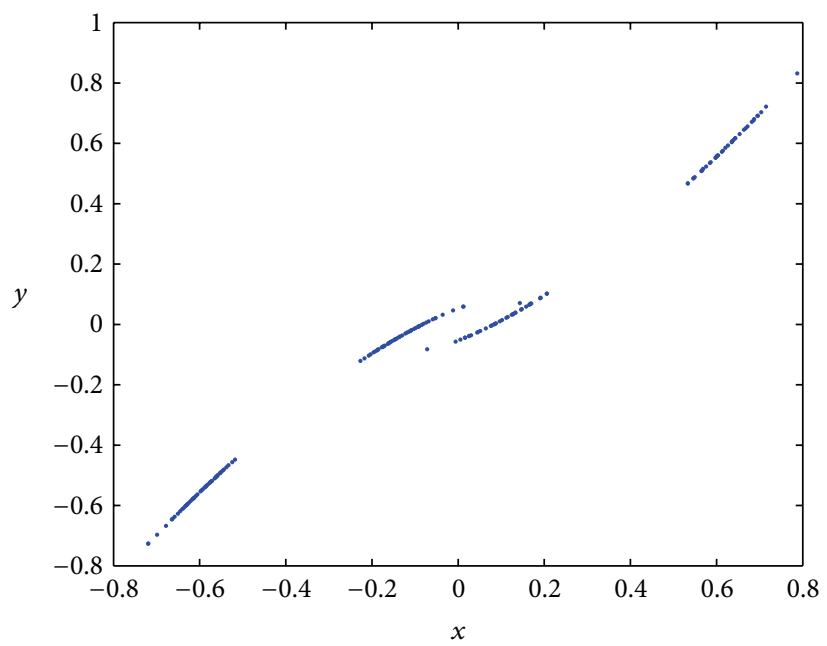

(c)

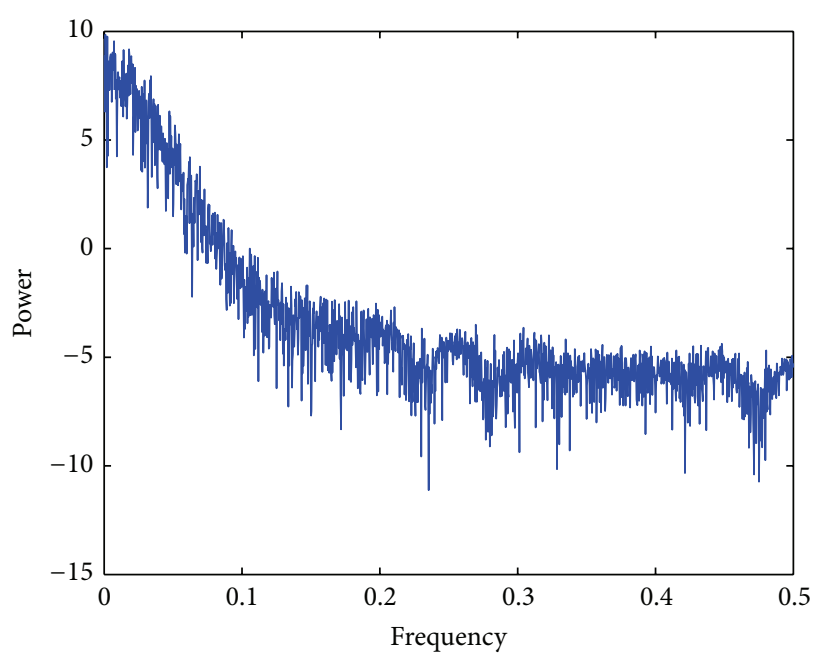

(b)

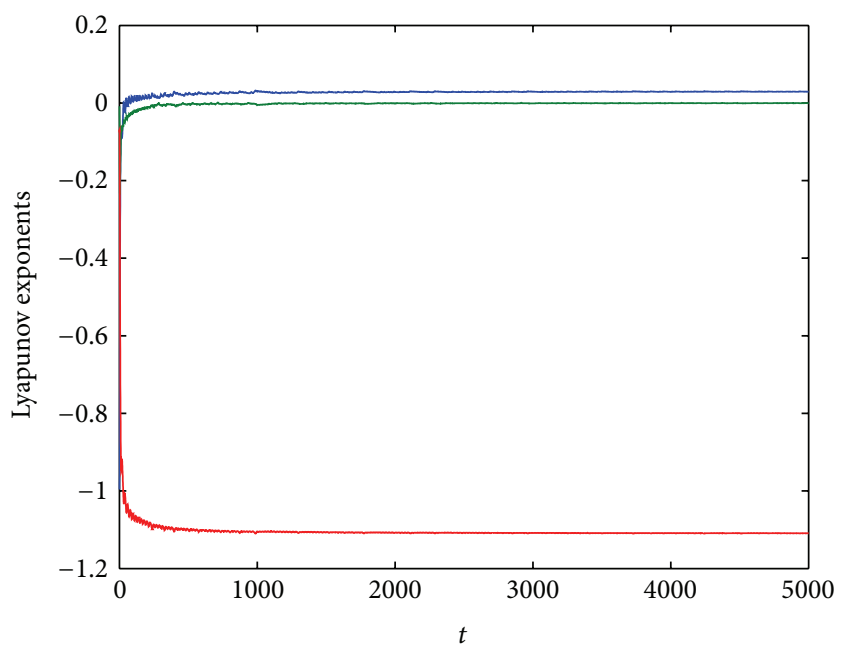

(d)

Figure 2: (a) Time history, (b) frequency spectrum, (c) Poincaré map in $x-y\{z=1.3\}$ plane, and (d) Lyapunov exponent spectrum of chaotic system (1).

where $\mathbf{A}=f_{x}\left(0, \zeta_{0}\right)$, and for $i=1,2,3,4$,

$$
\begin{gathered}
B_{i}(x, y)=\left.\sum_{j, k=1}^{4} \frac{\partial^{2} F_{i}(\xi)}{\partial \xi_{j} \partial \xi_{k}}\right|_{\xi=0} x_{j} y_{k}, \\
C_{i}(x, y, z)=\left.\sum_{j, k, l=1}^{4} \frac{\partial^{3} F_{i}(\xi)}{\partial \xi_{j} \partial \xi_{k} \partial \xi_{l}}\right|_{\xi=0} x_{j} y_{k} z_{l},
\end{gathered}
$$

and so on for $D_{i}$ and $E_{i}$.

Suppose that $\left(x_{0}, \zeta_{0}\right)$ is an equilibrium point of $(3)$, where the Jacobian matrix $\mathbf{A}$ has a pair of purely imaginary eigenvalues $\lambda_{2,3}= \pm i \omega_{0},\left(\omega_{0}>0\right)$, and admits no other eigenvalues with zero real part. Let $\mathbf{T}^{c}$ be the generalized eigenspace of $\mathbf{A}$ corresponding to $\lambda_{2,3}$. By this, the largest subspace invariant by $\mathbf{A}$ on which the eigenvalues are $\lambda_{2,3}$ is meant.
Let $p, q \in \mathbf{C}^{4}$ be vectors such that

$$
\mathbf{A} q=i \omega_{0} q, \quad \mathbf{A}^{T} p=-i \omega_{0} p, \quad\langle p, q\rangle=\sum_{i=1}^{4} \bar{p}_{i} q_{i}=1
$$

where $\mathbf{A}^{T}$ is the transpose of the matrix $\mathbf{A}$. Any vector $y \in \mathbf{T}^{c}$ can be represented as $y=\omega q+\bar{\omega} \bar{q}$, where $\omega=\langle p, y\rangle \in$ C. The two-dimensional center manifold associated with the eigenvalues $\lambda_{2,3}= \pm i \omega_{0}$ can be parameterized by the variables $\omega$ and $\bar{\omega}$ by means of an immersion of the form $x=H(\omega, \bar{\omega})$, where $H: \mathbf{C}^{2} \rightarrow \mathbf{R}^{4}$ has a Taylor expansion of the form

$$
H(\omega, \bar{\omega})=\omega q+\bar{\omega} \bar{q}+\sum_{2 \leq j+k \leq 5} \frac{1}{j ! k !} h_{j k} \omega^{j} \bar{\omega}^{k}+O\left(|\omega|^{6}\right)
$$


with $h_{j k} \in \mathbf{C}^{4}$ and $h_{j k}=\bar{h}_{k j}$. Substituting this expression into (3), we obtain the following differential equation:

$$
H_{\omega} \omega^{\prime}+H_{\bar{\omega}} \bar{\omega}^{\prime}=F(H(\omega, \bar{\omega})),
$$

where $F$ is given by (4). The complex vectors $h_{j k}$ are obtained solving the system of linear equations defined by the coefficients of (9), taking into account the coefficients of $F$, so that system (9), on the chart $\omega$ for a central manifold, writes as follows:

$$
\omega^{\prime}=i \omega_{0} \omega+\frac{1}{2} G_{21} \omega|\omega|^{2}+\frac{1}{12} G_{32} \omega|\omega|^{4}+O\left(|\omega|^{6}\right),
$$

with $G_{j k} \in \mathbf{C}$.

The first Lyapunov coefficient $l_{1}$ is defined by

$$
l_{1}=\frac{1}{2} \operatorname{Re} G_{21},
$$

where $G_{21}=\left\langle p, H_{21}\right\rangle, H_{21}=C(q, q, \bar{q})+B\left(\bar{q}, h_{20}\right)+2 B\left(q, h_{11}\right)$, $h_{20}=\left(2 i \omega_{0} \mathbf{I}_{4}-\mathbf{A}\right)^{-1} B(q, q), h_{11}=-\mathbf{A}^{-1} B(q, \bar{q})$, and $\mathbf{I}_{4}$ are unit $4 \times 4$ matrices.

The second Lyapunov coefficient is defined by

$$
l_{2}=\frac{1}{12} \operatorname{Re} G_{32}
$$

where $G_{32}=\left\langle p, H_{32}\right\rangle$,

$$
\begin{aligned}
H_{32}= & 6 B\left(h_{11}, h_{21}\right)+B\left(\bar{h}_{20}, h_{30}\right)+3 B\left(\bar{h}_{21}, \bar{h}_{20}\right) \\
& +3 B\left(q, h_{22}\right)+2 B\left(\bar{q}, h_{31}\right)+6 C\left(q, h_{11}, h_{11}\right) \\
& +3 C\left(q, \bar{h}_{20}, h_{20}\right)+3 C\left(q, q, \bar{h}_{21}\right)+6 C\left(q, \bar{q}, h_{21}\right) \\
& +6 C\left(\bar{q}, h_{20}, h_{11}\right)+C\left(\bar{q}, \bar{q}, h_{30}\right)+D\left(q, q, q, \bar{h}_{20}\right) \\
& +6 D\left(q, q, \bar{q}, h_{11}\right)+3 D\left(q, \bar{q}, \bar{q}, h_{20}\right) \\
& +E(q, q, q, \bar{q}, \bar{q})-6 G_{21} h_{21}-3 \bar{G}_{21} h_{21} .
\end{aligned}
$$

The complex vector $h_{21}$ can be found by solving the nonsingular $n+1$-dimensional system

$$
\left(\begin{array}{cc}
i \omega_{0} \mathbf{I}_{4}-\mathbf{A} & q \\
\bar{p} & 0
\end{array}\right)\left(\begin{array}{c}
h_{21} \\
s
\end{array}\right)=\left(\begin{array}{c}
H_{21}-G_{21} q \\
0
\end{array}\right)
$$

with the condition $\left\langle p, h_{21}\right\rangle=0$,

$$
\begin{gathered}
h_{30}=\left(3 i \omega_{0} \mathbf{I}_{4}-\mathbf{A}\right)^{-1}\left[3 B\left(q, h_{20}\right)+C(q, q, q)\right], \\
h_{31}=\left(2 i \omega_{0} \mathbf{I}_{4}-\mathbf{A}\right)^{-1}\left[3 B\left(q, h_{21}\right)+B\left(\bar{q}, h_{30}\right)+3 B\left(h_{20}, h_{11}\right)\right. \\
+3 C\left(q, q, h_{11}\right)+3 C\left(q, \bar{q}, h_{20}\right) \\
\left.+D(q, q, q, \bar{q})-3 G_{21} h_{20}\right], \\
h_{22}=-\mathbf{A}^{-1}\left[D(q, q, q, \bar{q})+4 C\left(q, \bar{q}, h_{11}\right)+C\left(\bar{q}, \bar{q}, h_{20}\right)\right. \\
+C\left(q, q, \bar{h}_{20}\right)+2 B\left(h_{11}, h_{11}\right)+2 B\left(q, \bar{h}_{21}\right) \\
\left.+2 B\left(\bar{q}, h_{21}\right)+B\left(\bar{h}_{20}, h_{20}\right)\right] .
\end{gathered}
$$

A Hopf point $\left(x_{0}, \zeta_{0}\right)$ of system (3) is an equilibrium point where, the Jacobian matrix $\mathbf{A}$ has a pair of purely imaginary eigenvalues $\lambda_{2,3}= \pm i \omega_{0},\left(\omega_{0}>0\right)$, and the other eigenvalue $\lambda_{1} \neq 0$. From the center manifold theorem, at a Hopf point, a two-dimensional center manifold is well defined it is invariant under the flow generated by (3) and can be continued with arbitrary high class of differentiability to the nearby parameter values.

A Hopf point is called transversal if the parameterdependent complex eigenvalues cross the imaginary axis with nonzero derivative. In a neighborhood of a transversal Hopf point with $l_{1} \neq 0$, the dynamic behavior of the system (3), reduced to the family of parameter-dependent continuations of the center manifold, is orbitally topologically equivalent to the following complex normal form: $\omega^{\prime}=\left(\eta+i \omega_{0}\right) \omega+l_{1} \omega|\omega|^{2}$, where $\omega \in \mathbf{C} . \eta$, $\omega$, and $l_{1}$ are real functions having derivatives of arbitrary higher order, which are continuations of $0, \omega_{0}$ and the first Lyapunov coefficient at the Hopf point. When $l_{1}<0\left(l_{1}>0\right)$, one family of stable (unstable) periodic orbits can be found on this family of manifolds, shrinking to an equilibrium point at the Hopf point.

A Hopf point of codimension 2 is a Hopf point, where $l_{1}$ vanishes. It is called transversal if $l_{1}=0$ and $\eta=0$ have transversal intersections, where $\eta=\eta(\mu)$ is the real part of the critical eigenvalues. In a neighborhood of a transversal Hopf point of codimension 2 with $l_{2} \neq 0$, the dynamic behavior of the system (3), reduced to the family of parameter-dependent continuations of the center manifold, is orbitally topologically equivalent to $\omega^{\prime}=\left(\eta+i \omega_{0}\right) \omega+\tau \omega|\omega|^{2}+l_{1} \omega|\omega|^{4}$, where $\eta$ and $\tau$ are unfolding parameters.

\section{Hopf Bifurcations at $E_{0}$}

In this section, we will study the stability of $E_{0}$ in the following controlled system:

$$
\begin{gathered}
\dot{x}=a(y-x), \\
\dot{y}=a(b x-x z)+u, \\
\dot{z}=-c z+x y, \\
\dot{v}=y-m v,
\end{gathered}
$$

where $u=k_{1}(y-m v)+k_{2}(y-m v)^{3}, k_{1}, k_{2}$ are the control gain vectors, and $m$ is the washout-filter time constant. Obviously, the controller $u$ keeps the equilibrium structure and does not change the divergence of the dynamic system (1).

The Jacobian matrix of the system (16) at $E_{0}$ is given by

$$
\mathbf{A}=\left(\begin{array}{cccc}
-a & a & 0 & 0 \\
a b & k_{1} & 0 & -m k_{1} \\
0 & 0 & -c & 0 \\
0 & 1 & 0 & -m
\end{array}\right)
$$

The characteristic polynomial of the Jacobian matrix of system (16) at $E_{0}$ has the form

$$
\begin{aligned}
p(\lambda)=(\lambda+c)[ & \lambda^{3}+\left(a+m-k_{1}\right) \lambda^{2} \\
& \left.+\left(a m-a k_{1}-a^{2} b\right) \lambda+a^{2} b m\right] .
\end{aligned}
$$




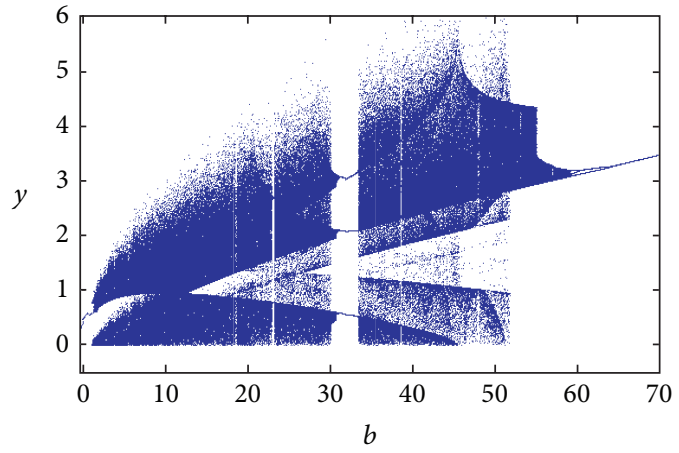

(a)

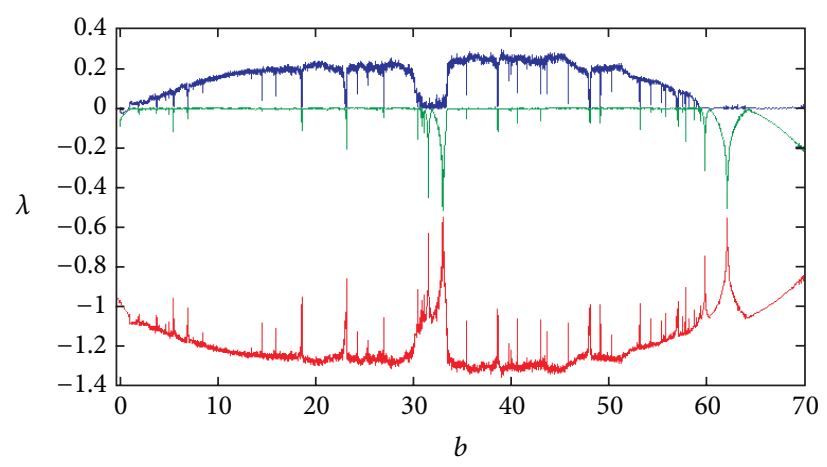

(b)

Figure 3: Nonlinear dynamics of system (1) for specific values $a=1, c=0.08$ versus the control parameter $b$. (a) Bifurcation diagram of $y$. (b) Lyapunov exponent spectrum.

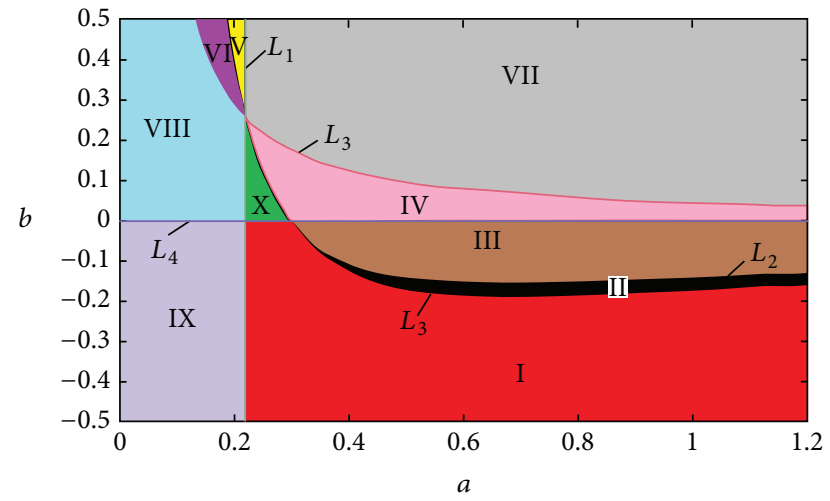

FIGURE 4: The stable domain on the parameter plane $(a, b)$ with $c=$ 0.08 , and $k_{1}=0.8, k_{2}=0.5$.

If $c>0, a+m-k_{1}>0, a^{2} b m<0$, and

$$
b<b_{0}=\frac{m^{2}+k_{1}^{2}-2 m k_{1}+a m-a k_{1}}{a^{2}+2 a m-a k_{1}},
$$

then the equilibrium $E_{0}$ is asymptotically stable. If $b>b_{0}$, the equilibrium $E_{0}$ is unstable.

The equation $b=b_{0}$ in (19) gives the equation of the Hopf hypersurfaces in the parameter space $\left(a, m, k_{1}\right)$. This equation will be used in this Section in the study of Hopf bifurcations which occur at the equilibria $E_{0}$ of system (16).

Then, using the notion of the previous section, the multilinear symmetric functions corresponding to $f$ can be written as

$$
\begin{gathered}
B(x, y)=\left(0,-a\left(x_{1} y_{3}+x_{3} y_{1}\right), x_{1} y_{2}+x_{2} y_{1}, 0\right)^{T}, \\
C(x, y, z)=\left(0,6 k_{2}\left(x_{2} y_{2} z_{2}-m^{3} x_{4} y_{4} z_{4}+m^{2} x_{2} y_{4} z_{4}\right.\right. \\
+m^{2} x_{4} y_{2} z_{4}+m^{2} x_{4} y_{4} z_{2}-m x_{2} y_{2} z_{4} \\
\left.\left.-m x_{2} y_{4} z_{2}-m x_{4} y_{2} z_{2}\right), 0,0\right)^{T} .
\end{gathered}
$$

The eigenvalues of $\mathbf{A}$ are

$$
\begin{aligned}
\lambda_{1,2} & = \pm i \omega_{0}= \pm i \sqrt{a m-a k_{1}-a^{2} b}, \\
\lambda_{3} & =-c, \quad \lambda_{4}=-\left(a+m-k_{1}\right) .
\end{aligned}
$$

And from (7), one has

$$
\begin{gathered}
q=\left(\frac{a\left(a m+\omega_{0}^{2}\right)+a \omega_{0}(a-m) i}{a^{2}+\omega_{0}^{2}}, m+\omega_{0} i, 0,1\right)^{T}, \\
p=\left(p_{1}+p_{2} i, p_{3}+p_{4} i, 0, p_{5} i\right)^{T},
\end{gathered}
$$

where

$$
\begin{gathered}
\delta_{1}=\left(a^{2}+\omega_{0}^{2}\right)\left(m^{2}-\omega_{0}^{2}+m k_{1}\right)-\left(2 k_{1} m+\omega_{0}^{2}\right)\left(a m+\omega_{0}^{2}\right) \\
-\omega_{0}^{2}\left(m-k_{1}\right)(a-m), \\
\delta_{2}=2 m \omega_{0}\left(a^{2}+\omega_{0}^{2}\right)-\omega_{0}\left(m-k_{1}\right)\left(a m+\omega_{0}^{2}\right) \\
\quad-\omega_{0}(a-m)\left(2 k_{1} m+\omega_{0}^{2}\right), \\
p_{1}=\frac{\left(a^{2}+\omega_{0}^{2}\right)\left[\delta_{2} \omega_{0}\left(m-k_{1}\right)-\delta_{1}\left(2 k_{1} m-\omega_{0}^{2}\right)\right]}{a\left(\delta_{1}^{2}+\delta_{2}^{2}\right)}, \\
p_{3}=\frac{\left(a^{2}+\omega_{0}^{2}\right)\left(\delta_{1} m+\delta_{2} \omega_{0}\right)}{a\left(\delta_{1}^{2}+\delta_{2}^{2}\right)}, \\
p_{2}=\frac{\left(a^{2}+\omega_{0}^{2}\right)\left[-\delta_{2}\left(2 k_{1} m-\omega_{0}^{2}\right)-\delta_{1} \omega_{0}\left(m-k_{1}\right)\right]}{a\left(\delta_{1}^{2}+\delta_{2}^{2}\right)}, \\
p_{4}=\frac{\left(a^{2}+\omega_{0}^{2}\right)\left(\delta_{1} \omega_{0}-\delta_{2} m\right)}{a\left(\delta_{1}^{2}+\delta_{2}^{2}\right)},
\end{gathered}
$$




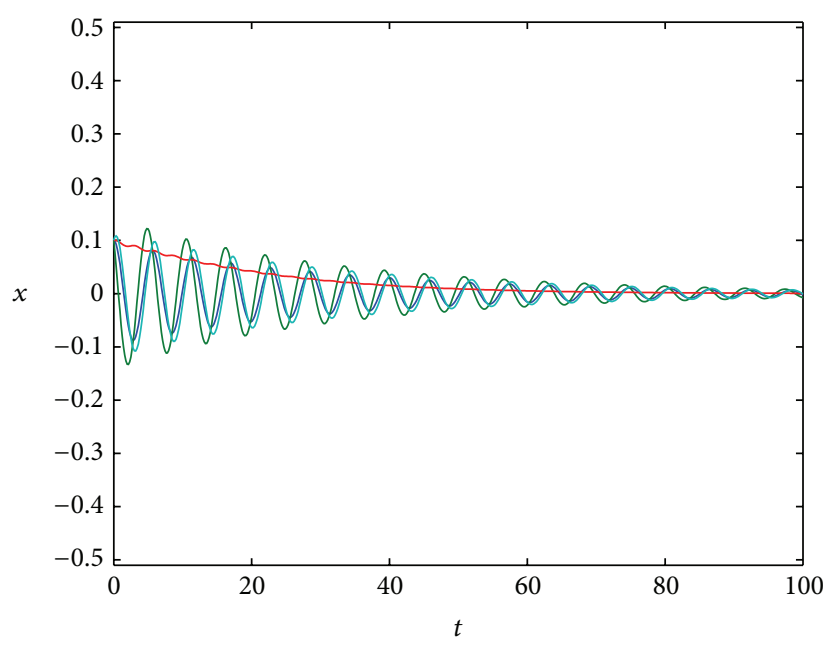

(a)

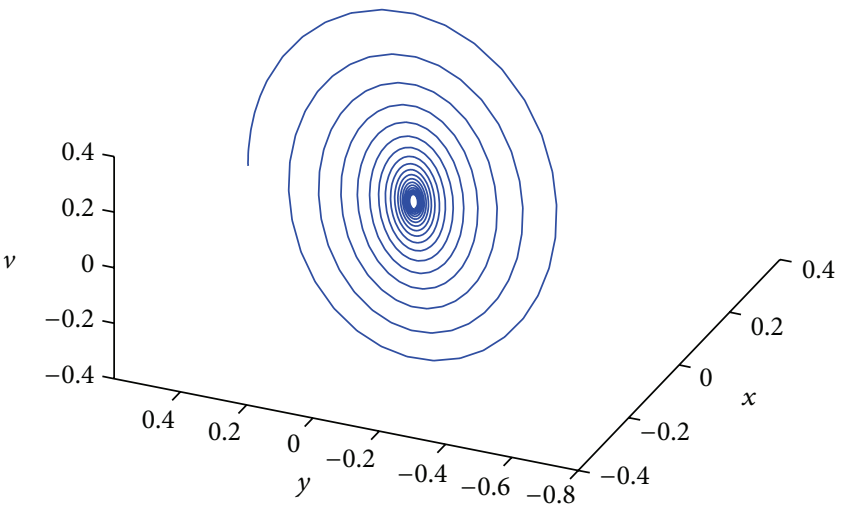

(b)

Figure 5: Time history and phase diagram of system (16) with $a=1, c=0.08, m=0.5, k_{1}=0.8, k_{2}=0.5$, and $b=-0.2$.

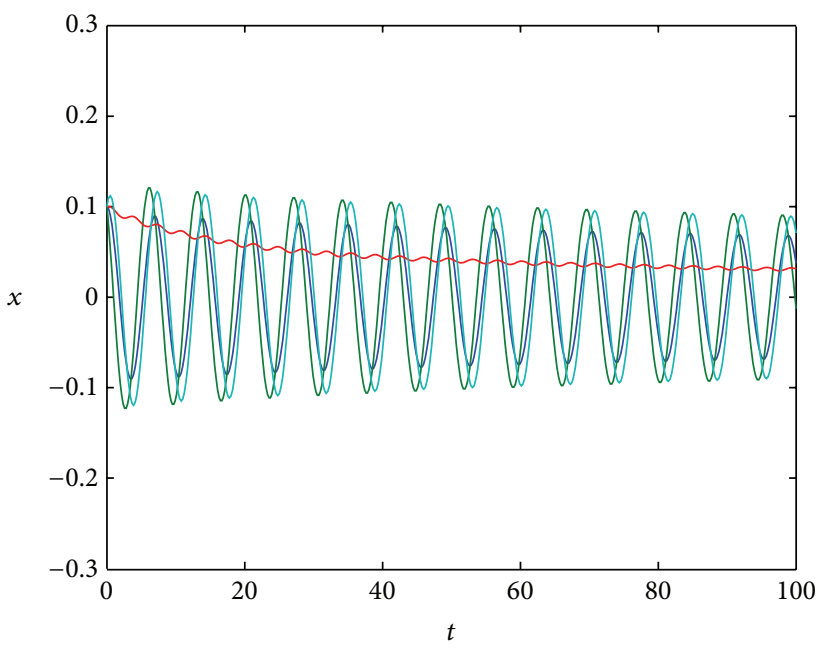

(a)

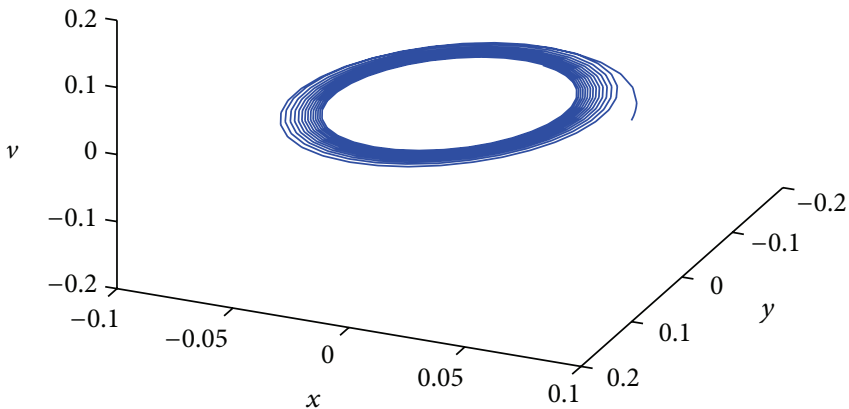

(b)

FIGURE 6: Time history and phase diagram of system (16) with $a=1, c=0.08, m=0.5, k_{1}=0.8, k_{2}=0.5$, and $b=-0.175$.

$$
p_{5}=\frac{m k_{1}\left(a^{2}+\omega_{0}^{2}\right)\left(\delta_{1}+\delta_{2}\right)}{a\left(\delta_{1}^{2}+\delta_{2}^{2}\right)}
$$

$B(q, q)$

$=\left(0, \frac{2 a\left[\left(a \omega_{0}^{2}-a m^{2}-2 m \omega_{0}^{2}\right)+\left(m^{2} \omega_{0}+2 a m \omega_{0}-\omega_{0}^{3}\right) i\right]}{a^{2}+\omega_{0}^{2}}\right.$,

$0,0)^{T}$

$B(q, \bar{q})=\left(0, \frac{2 a\left(m^{2}+\omega_{0}^{2}\right)}{a^{2}+\omega_{0}^{2}}, 0,0\right)^{T}$,

$$
\begin{gathered}
h_{11}=\left(\frac{-2 a\left(m^{2}+\omega_{0}^{2}\right)}{b\left(a^{2}+\omega_{0}^{2}\right)}, \frac{-2 a\left(m^{2}+\omega_{0}^{2}\right)}{b\left(a^{2}+\omega_{0}^{2}\right)}, 0, \frac{-2 a\left(m^{2}+\omega_{0}^{2}\right)}{b m\left(a^{2}+\omega_{0}^{2}\right)}\right)^{T}, \\
h_{20}=\left(2 i \omega_{0} I_{3}-A\right)^{-1} B(q, q)=\left(h_{1}, h_{2}, 0, h_{3}\right)^{T}
\end{gathered}
$$

where

$$
\begin{gathered}
\delta_{3}=\left(c i-2 \omega_{0}\right)\left(2 a m \omega_{0}-8 \omega_{0}^{3}-2 a k_{1} \omega_{0}-4 k_{1} \omega_{0}^{2} i\right. \\
\left.-2 a^{2} b \omega_{0}+4 m \omega_{0}^{2} i+4 a \omega_{0}^{2} i+a^{2} b m i\right), \\
\delta_{4}=a\left(-2 \omega_{0}+m i\right)\left(8 \omega_{0}^{3}-4 k_{1} \omega_{0}^{2} i+2 a k_{1} \omega_{0} i\right. \\
+2 a k_{1} \omega_{0}-2 a m \omega_{0}+2 a^{2} b \omega_{0} \\
\left.+4 m \omega_{0}^{2} i+4 a \omega_{0}^{2} i+a^{2} b m i\right)
\end{gathered}
$$




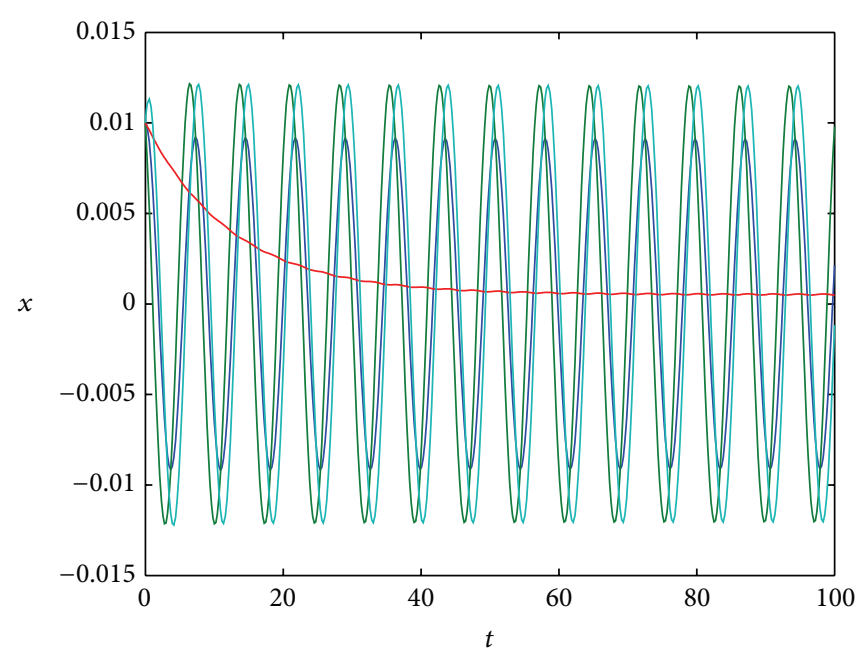

(a)

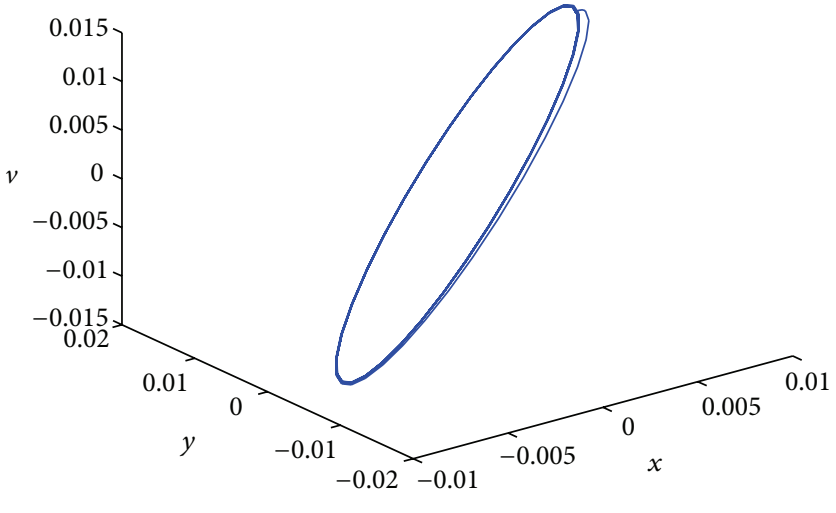

(b)

FIGURE 7: Time history and phase diagram of system (16) with $a=1, c=0.08, m=0.5, k_{1}=0.8, k_{2}=0.5$, and $b=-0.15$.

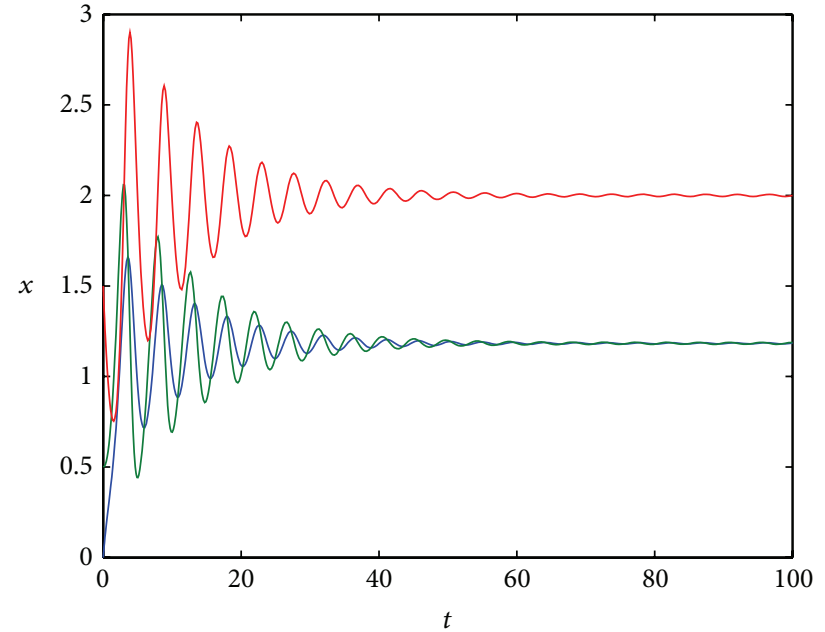

(a)

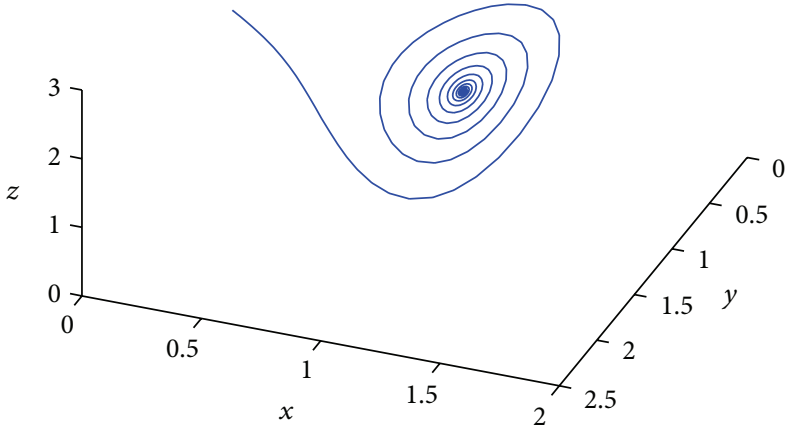

(b)

FIgURE 8: Time history and phase diagram of system (1) with $a=1, b=2$, and $c=0.6998$.

$$
\begin{aligned}
\delta_{5}= & a^{2} b^{2} m^{2}+4 a^{2} b^{2} \omega_{0}^{2}+8 a^{2} b k_{1} \omega_{0}^{2}-8 a^{2} b k_{1} m \omega_{0}^{2} \\
& +8 a^{2} b m^{2} \omega_{0}^{2}+32 a^{2} b \omega_{0}^{4}+4 a^{2} k_{1}^{2} \omega_{0}^{2} \\
& -8 a^{2} k_{1} m \omega_{0}^{2}+4 a^{2} m^{2} \omega_{0}^{2}+16 a^{2} \omega_{0}^{2} \\
& +16 k_{1}^{2} \omega_{0}^{4}-32 k_{1} m \omega_{0}^{4}+16 m^{2} \omega_{0}^{4}+64 \omega_{0}^{6}, \\
h_{1}= & \left(2 a \delta _ { 4 } \left[\left(a \omega_{0}^{2}-a m^{2}-2 m \omega_{0}^{2}\right)\right.\right. \\
& \left.\left.\quad+\left(m^{2} \omega_{0}+2 a m \omega_{0}-\omega_{0}^{3}\right) i\right]\right) \\
& \times\left(\delta_{5}\left(a^{2}+\omega_{0}^{2}\right)\right)^{-1}
\end{aligned}
$$

$$
\begin{aligned}
h_{2}=( & \left(a+\omega_{0}\right)\left(c+2 \omega_{0} i\right)\left(m+2 \omega_{0} i\right) \\
\times & {\left.\left[\left(a \omega_{0}^{2}-a m^{2}-2 m \omega_{0}^{2}\right)+\left(m^{2} \omega_{0}+2 a m \omega_{0}-\omega_{0}^{3}\right) i\right]\right) } \\
& \times\left(\delta_{3}\left(a^{2}+\omega_{0}^{2}\right)\right)^{-1}, \\
h_{3}=\left(2\left(c+2 \omega_{0} i\right)\left(a+2 \omega_{0} i\right)\right. & \left.\times\left[\left(a \omega_{0}^{2}-a m^{2}-2 m \omega_{0}^{2}\right)+\left(m^{2} \omega_{0}+2 a m \omega_{0}-\omega_{0}^{3}\right) i\right]\right) \\
& \times\left(\delta_{3}\left(a^{2}+\omega_{0}^{2}\right)\right)^{-1} .
\end{aligned}
$$




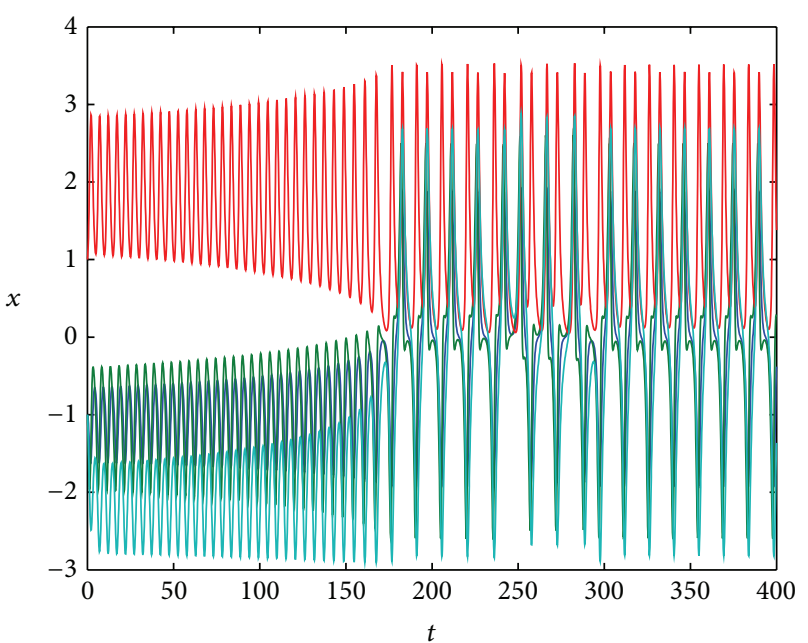

(a)

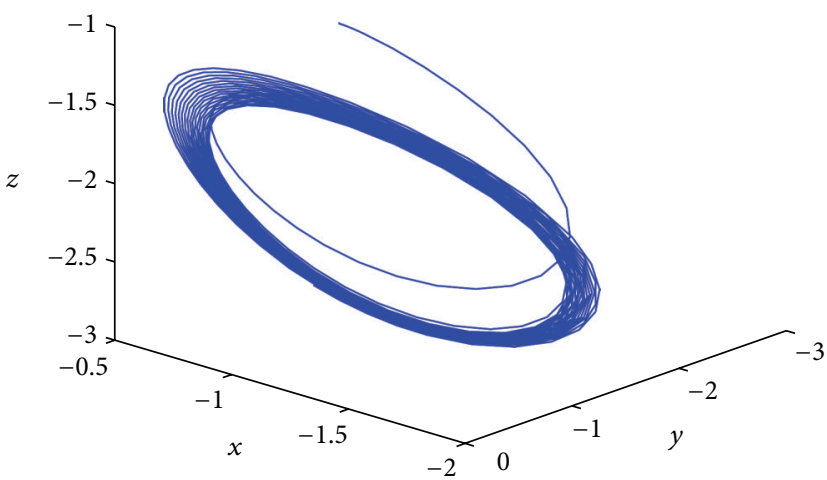

(b)

Figure 9: Time history and phase diagram of system (16) with $a=1, b=2$, and $c=0.6998$.

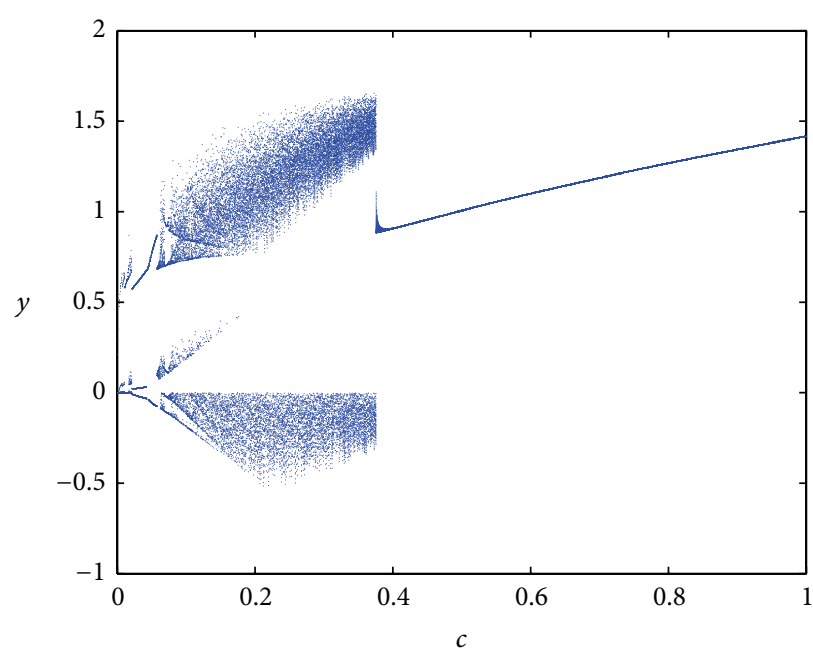

(a)

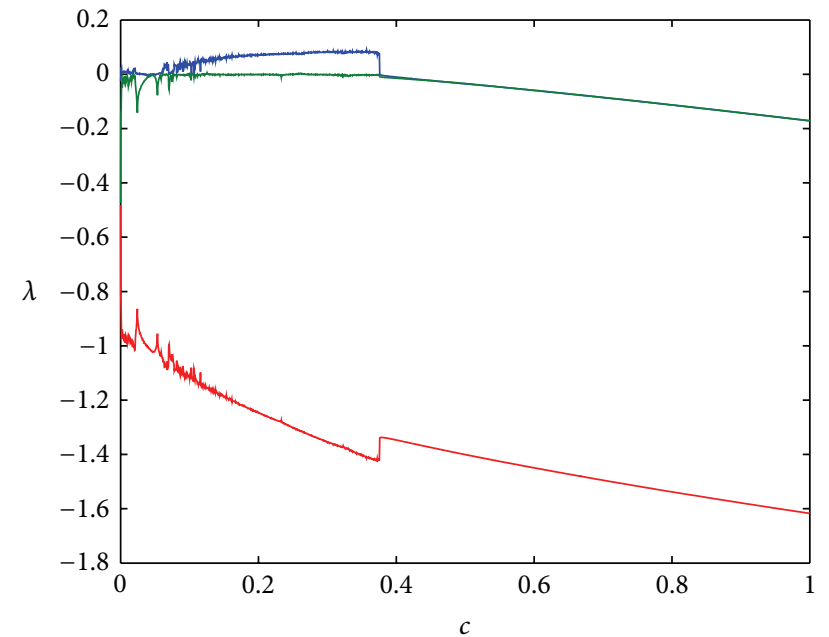

(b)

FIGURE 10: Nonlinear dynamics of system (1) for specific values $a=1, b=2$ versus the control parameter $c$. (a) Bifurcation diagram of $y$. (b) Lyapunov exponent spectrum.

One also has

$$
\begin{gathered}
B\left(q, h_{11}\right)=\left(0,0,\left(\left(-2 a\left(m^{2}+\omega_{0}^{2}\right)\right.\right.\right. \\
\times\left[a \omega_{0}^{2}+m 2 a^{2}+m \omega_{0}^{2}\right. \\
\left.\left.+\omega_{0}\left(2 a^{2}+\omega_{0}^{2}-a m\right) i\right]\right) \\
\left.\left.\times\left(b\left(a^{2}+\omega_{0}^{2}\right)^{2}\right)^{-1}\right), 0\right)^{T}, \\
B\left(\bar{q}, h_{20}\right)=\left(0,0,\left(\left(a h_{2}\left[\left(a m+\omega_{0}^{2}\right)-\omega_{0}(a-m) i\right]\right.\right.\right. \\
\left.+h_{1}\left(a^{2}+\omega_{0}^{2}\right)\left(m-\omega_{0} i\right)\right) \\
\left.\left.\times\left(a^{2}+\omega_{0}^{2}\right)^{-1}\right), 0\right)^{T}
\end{gathered}
$$

$$
\begin{gathered}
C(q, q, \bar{q})=\left(0, h_{4}+h_{5} i, 0,0\right)^{T}, \\
H_{21}=\left(0, h_{4}+h_{5} i, h_{6}+h_{7} i, 0\right)^{T}, \\
G_{21}=\left(h_{4} p_{3}-h_{5} p_{4}\right)+\left(h_{5} p_{3}+h_{4} p_{4}\right) i,
\end{gathered}
$$

where

$$
\begin{aligned}
& h_{4}= 6 m k_{2}\left[(m+1)\left(\omega_{0}^{2}+3 m^{2}\right)-\left(3 m^{2}+2 \omega_{0}^{2}\right)\right], \\
& h_{5}=6 k_{2} \omega_{0}^{3}, \\
& h_{6}=a b\left(a m+\omega_{0}^{2}\right)+h_{1} b m\left(a^{2}+\omega_{0}^{2}\right) \\
&-4 a\left(m^{2}+\omega_{0}^{2}\right)\left[a\left(a m+\omega_{0}^{2}\right)+m\left(a^{2}+\omega_{0}^{2}\right)\right],
\end{aligned}
$$




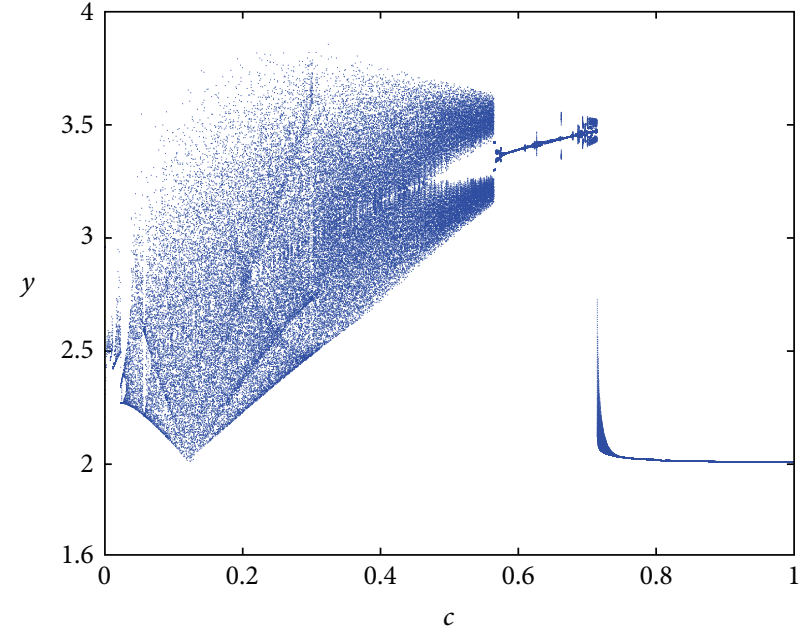

(a)

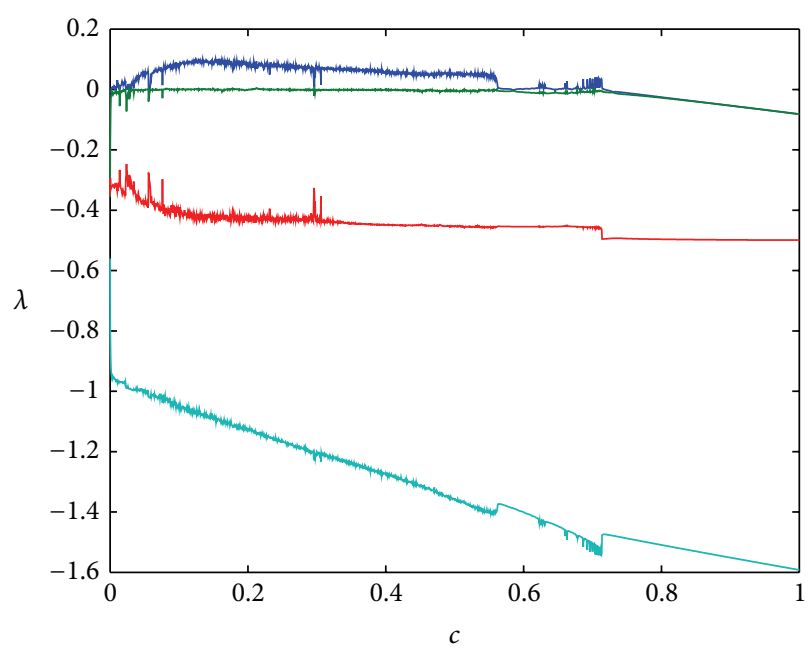

(b)

FIGURE 11: Nonlinear dynamics of controlled system (16) for specific values $a=1, b=2$ versus the control parameter $c$. (a) Bifurcation diagram of $y$. (b) Lyapunov exponent spectrum.

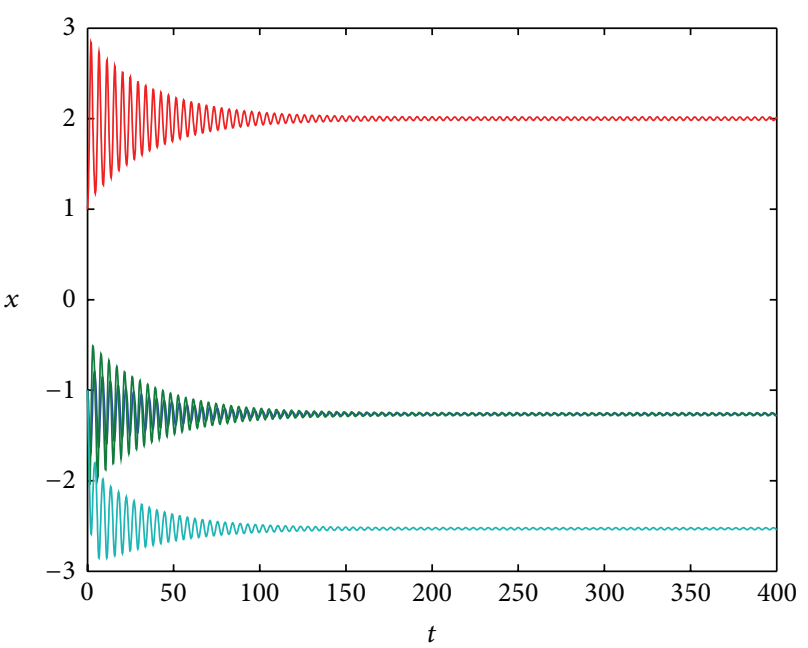

(a)

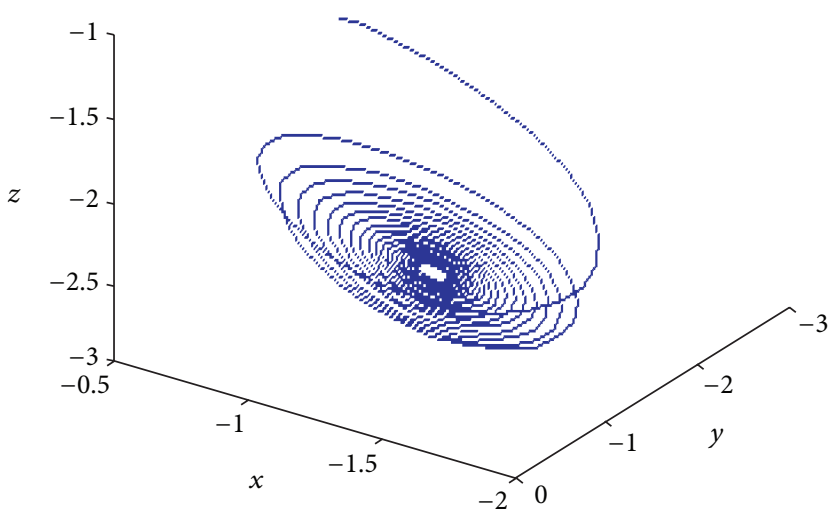

(b)

Figure 12: Time history and phase diagram of system (16) with $a=1, b=2, m=0.5, k_{1}=0.2, k_{2}=0.5$, and $c=0.8$.

$$
\begin{aligned}
h_{7}= & -a b \omega_{0}(a-m)-h_{1} b \omega_{0}\left(a^{2}+\omega_{0}^{2}\right) \\
& -4 a \omega_{0}\left(m^{2}+\omega_{0}^{2}\right)\left[a\left(a m+\omega_{0}^{2}\right)+m\left(a^{2}+\omega_{0}^{2}\right)\right] .
\end{aligned}
$$

Now, consider the family of differential equation (16) regarded as dependent on the parameter $b$. The real part $\xi$ of the pair of complex eigenvalues at the critical parameter $b=b_{0}$ verifies

$$
\begin{aligned}
\xi^{\prime}\left(b_{0}\right) & =\operatorname{Re}\left\langle p,\left.\frac{d \mathbf{A}}{d b}\right|_{b=b_{0}} q\right\rangle \\
& =p_{3}\left(a m+\omega_{0}^{3}\right)-p_{4} \omega_{0}(a-m) .
\end{aligned}
$$

If $\xi^{\prime}\left(b_{0}\right) \neq 0$, the transversality condition at the Hopf point holds.

Using these calculations, we prove the next theorem.

Theorem 1. Consider the six-parameter family of differential equations (16). The first Lyapunov coefficient associated with the equilibrium $E_{0}$ is given by

$$
l_{1}=\frac{1}{2}\left(h_{4} p_{3}-h_{5} p_{4}\right)
$$

If $l_{1}$ is different from zero and the transversality condition at the Hopf point holds, then system (16) has a transversal Hopf point at $E_{0}$. 


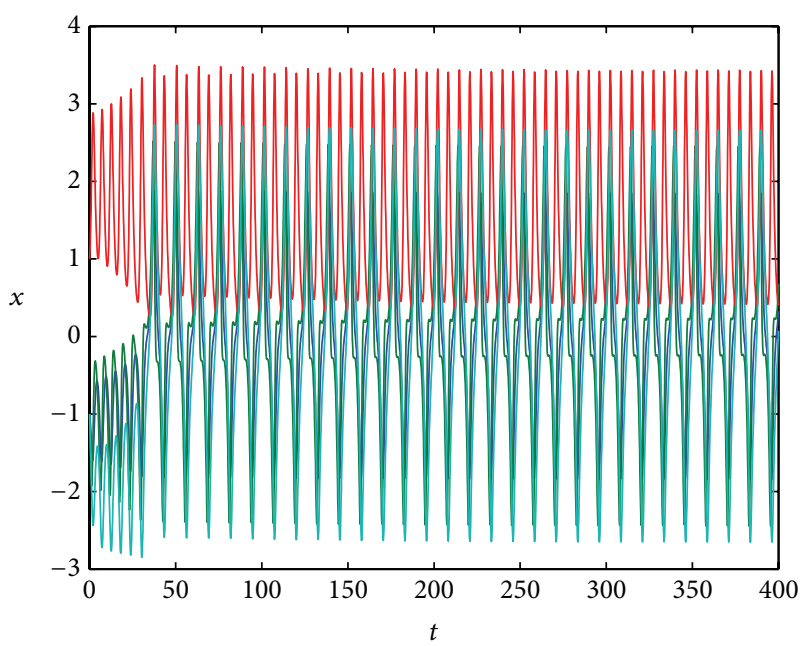

(a)

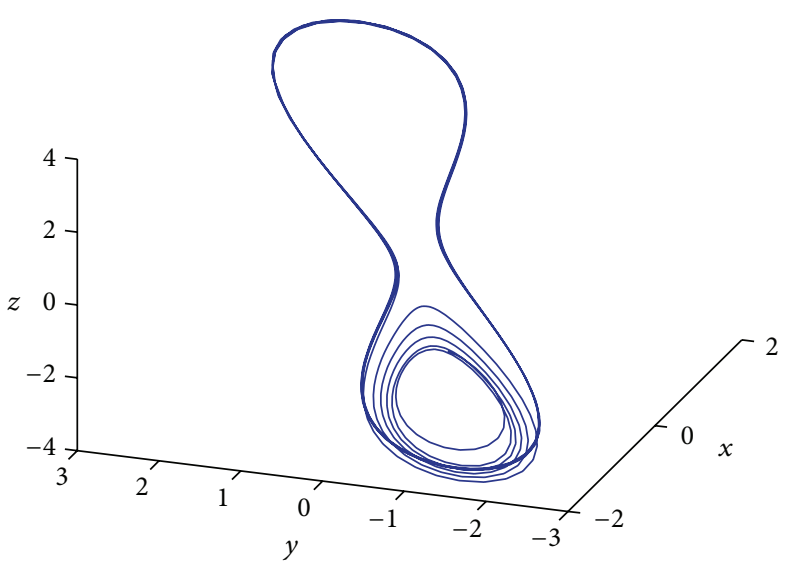

(b)

FIGURE 13: Time history and phase diagram of system (16) with $a=1, b=2, m=0.5, k_{1}=0.2, k_{2}=0.5$, and $c=0.65$.

Let the damping coefficient $m=0.5$. The washout filter is designed as

$$
u=k_{1}(y-0.5 v)+k_{2}(y-0.5 v)^{3} \text {. }
$$

If $0<k_{1}<1$, then the system (16) has a transversal Hopf point at $E_{0}$, when $a=1, c=0.08$. More specifically, if $k_{2}>0$, there exists a stable periodic orbit near the unstable equilibrium point $E_{0}$; if $k_{2}<0$, there exists an unstable periodic orbit near the asymptotically stable equilibrium point $E_{0}$.

When the parameters $a=1$ and $c=0.08$ are fixed, while parameter $b$ is varied in the interval $[-0.5,70]$, some different dynamical behaviors of system (1) are obtained. The bifurcation diagram of system (1) in terms of the parameter $b$ is depicted in Figure 3(a). Figure 3(b) shows the corresponding Lyapunov exponent spectrum versus the increasing $b$. As $b$ increases, system (1) is undergoing the following dynamical routes:

(1) if $-0.5<b \leq 0.95$, the system is stable,

(2) if $0.95<b \leq 31$, the system is chaotic. But there are two periodic windows in the chaotic band,

(3) if $31<b \leq 33.4$, there is a reverse period-three bifurcation route with a flip bifurcation,

(4) if $33.4<b \leq 59.2$, the system is chaotic. But there are several periodic windows in the chaotic band,

(5) if $59.2<b \leq 70$, there is a very long reverse perioddoubling bifurcation window.

With the analysis performed here, one can find that the Hopf bifurcation at the equilibrium $E_{0}$ does not occur when $b \in[-0.5,70]$. We design a control laws such that our feedback system (16) undergoes a controllable Hopf bifurcation when the parameter $b=-0.175$, as shown in Figures 4-7.

According to Routh-Hurwitz criterion, if and only if (30) is satisfied, then the equilibrium $E_{0}$ is asymptotically stable

$$
\begin{gathered}
\Delta_{1}=p_{0}>0, \quad \Delta_{2}=\left|\begin{array}{cc}
p_{0} & 1 \\
p_{2} & p_{1}
\end{array}\right|>0, \\
\Delta_{3}=\left|\begin{array}{ccc}
p_{0} & 1 & 0 \\
p_{2} & p_{1} & p_{0} \\
0 & p_{3} & p_{2}
\end{array}\right|>0, \quad \Delta_{4}=p_{3} \Delta_{3}>0,
\end{gathered}
$$

where

$$
\begin{gathered}
p_{0}=a+m-k_{1}, \\
p_{1}=a m-a k_{1}-a^{2} b+a c+m a-c k_{1}, \\
p_{2}=a^{2} b m+a m c-a c k_{1}-a^{2} b c \\
p_{3}=a^{2} b m c .
\end{gathered}
$$

If $\Delta_{3}=0, \Delta_{i}>0, i=1,2,4$, then the characteristic polynomial of the Jacobian matrix of system (16) at $E_{0}$ with one pair of conjugate nonzero purely imaginary eigenvalues and the real part of other characteristic roots are less than 0 .

Let $\Delta_{1}=0$, let $\Delta_{2}=0$, let $\Delta_{3}=0$, and let $\Delta_{4}=0$, using MATLAB to draw the stability region on the parameter plane $a-b$, as shown in Figure 4 . In the figure, the symbol $L_{i}, i=1,2,3,4$, represents $\Delta_{i}=0, i=1,2,3,4$. And in the region (I): $\Delta_{1}>0, \Delta_{2}>0, \Delta_{3}>0$, and $\Delta_{4}>0$, in the region (II): $\Delta_{1}>0, \Delta_{2}>0, \Delta_{3}>0$, and $\Delta_{4}<0$, in the region (III): $\Delta_{1}>0, \Delta_{2}<0, \Delta_{3}>0$, and $\Delta_{4}<0$, in the region (IV): $\Delta_{1}>0, \Delta_{2}<0, \Delta_{3}>0$, and $\Delta_{4}>0$, in the region (V): $\Delta_{1}<0, \Delta_{2}<0, \Delta_{3}<0$, and $\Delta_{4}<0$, in the region (VI): $\Delta_{1}<0, \Delta_{2}>0, \Delta_{3}>0$, and $\Delta_{4}>0$, in the region (VII): $\Delta_{1}>0, \Delta_{2}<0, \Delta_{3}<0$, and $\Delta_{4}<0$, in the region (VIII): 


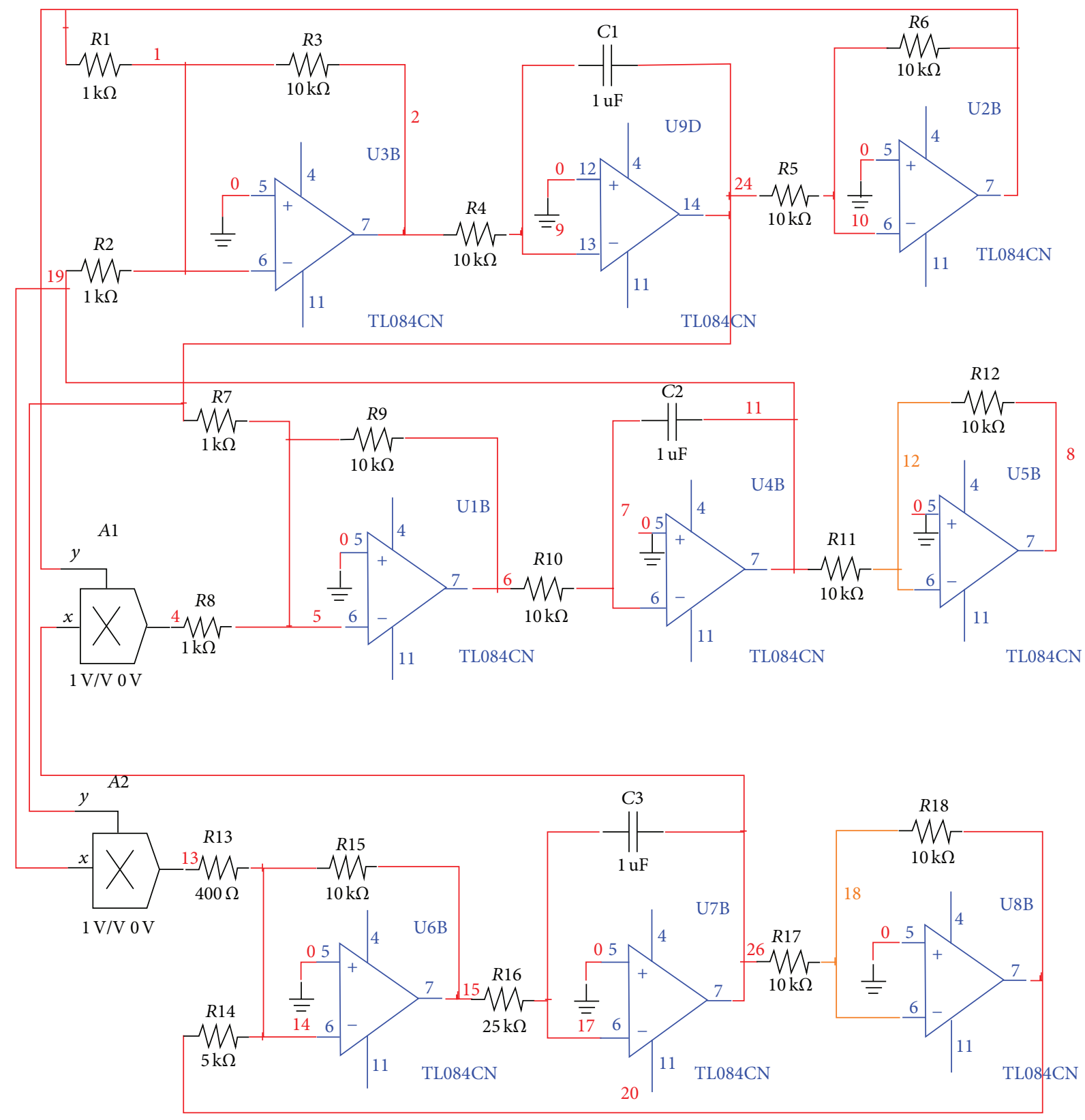

FIGURE 14: Circuit diagram for realizing the chaotic attractor of system.

$\Delta_{1}<0, \Delta_{2}>0, \Delta_{3}<0$, and $\Delta_{4}<0$, in the region (IX): $\Delta_{1}<0, \Delta_{2}>0, \Delta_{3}<0$, and $\Delta_{4}>0$, and in the region (X): $\Delta_{1}>0, \Delta_{2}>0, \Delta_{3}<0$, and $\Delta_{4}<0$. All the points are stable in the region (I).

Next, we combined with Figure 4 and let $a=1, c=0.08$, $k_{1}=0.8$, and $k_{2}=0.5$; then, we can compute the Hopf bifurcation value $b_{0}=-0.175$. The equilibrium is stable when $b=-0.2<b_{0}$ and unstable when $b=-0.15>b_{0}$, as shown in Figures 5 and 7, respectively. The system (16) has a transversal Hopf point at $E_{0}$ when $b=b_{0}=-0.175$ as shown in Figure 6. From the formulas in previous sections, we have $l_{1}=$ $-2.8234<0, \xi^{\prime}\left(b_{0}\right)=-1.0719<0$. Thus, the periodic solution bifurcating from $E_{0}$ is supercritical and stable.

\section{Hopf Bifurcations at $E_{+}$}

In this section, we will study the stability of $E_{+}$in the controlled system (16). The Jacobian matrix of the system (16) at $E_{+}$is given by

$$
\mathbf{A}=\left(\begin{array}{cccc}
-a & a & 0 & 0 \\
0 & k_{1} & -\sqrt{b c} & -m k_{1} \\
\sqrt{b c} & \sqrt{b c} & -c & 0 \\
0 & 1 & 0 & -m
\end{array}\right)
$$

The characteristic polynomial of the Jacobian matrix of system (16) at $E_{+}$has the form

$$
p(\lambda)=\lambda^{4}+A \lambda^{3}+B \lambda^{2}+C \lambda+D,
$$




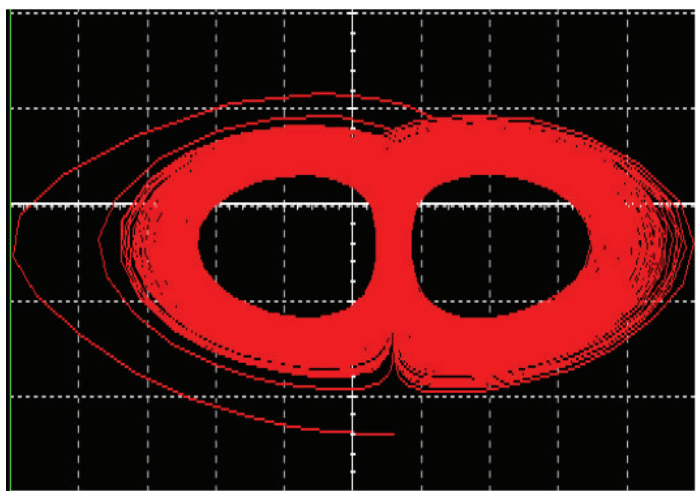

(a) Projection on $y$ - $z$ plane

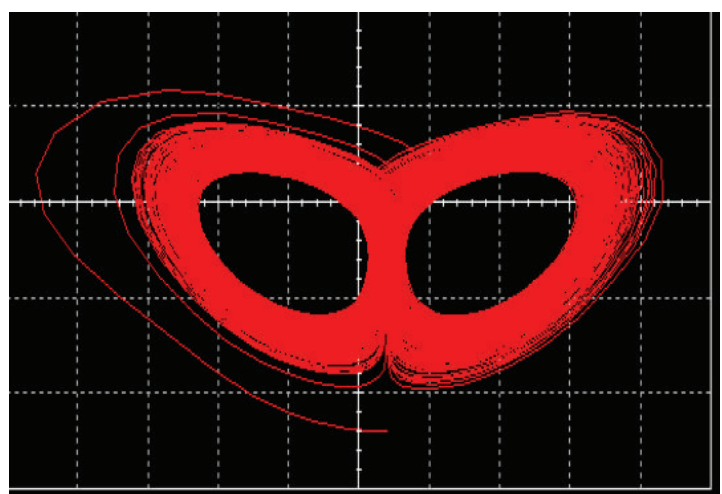

(b) Projection on $x$ - $z$ plane

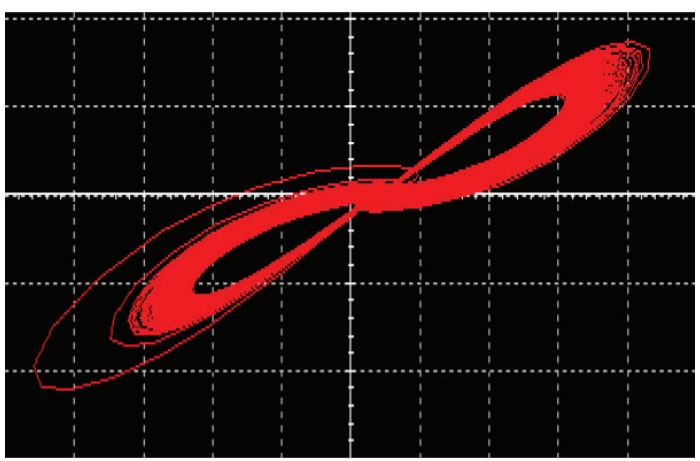

(c) Projection on $x-y$ plane

FIGURE 15: Experimental observations of the chaotic attractor in different planes.

where

$$
\begin{gathered}
A=a+m+c-k_{1}, \\
B=a b c+m c-c k_{1}+a m+a c-a k, \\
C=a^{2} b c-a c k_{1}+m a c+m a b c+a b c, \\
D=2 a^{2} b c m .
\end{gathered}
$$

If $A>0, B>0, C>0, D>0$, and

$$
C(C-A B)+A^{2} D=0,
$$

then system (16) has a transversal Hopf point at $E_{+}$.

According to Dias et al. [11], the equilibrium $E_{+}$is locally asymptotically stable when $(a, b, c) \in v$. In this section, the washout filter is designed as

$$
u=k_{1}(y-0.5 v)+k_{2}(y-0.5 v)^{3},
$$

to realize Hopf bifurcation at $E_{+}$in the parameter space $(a, b, c) \in v$. We fixed $a=1, b=2$, and $c=0.6998>$ $a(b-1) /(b+1)$ and then study the Hopf bifurcation at $E_{+}$in the controlled system (16). Assuming that the controlled system (16) undergoes a Hopf bifurcation at the equilibrium $E_{+}$, then (35) is satisfied. By solving (35), we can get $k_{1}=0.2$.
Then, using the notion of the previous section, the multilinear symmetric functions corresponding to $f$ can be written as

$$
\begin{gathered}
B(x, y)=\left(0,-x_{1} y_{3}-x_{3} y_{1}, x_{1} y_{2}+x_{2} y_{1}, 0\right)^{T} \\
C(x, y, z)=\left(0,6 k_{2}\left(x_{2} y_{2} z_{2}-m^{3} x_{4} y_{4} z_{4}+m^{2} x_{2} y_{4} z_{4}\right.\right. \\
+m^{2} x_{4} y_{2} z_{4}+m^{2} x_{4} y_{4} z_{2}-m x_{2} y_{2} z_{4} \\
\left.\left.-m x_{2} y_{4} z_{2}-m x_{4} y_{2} z_{42}\right), 0,0\right)^{T}
\end{gathered}
$$

The eigenvalues of $\mathbf{A}$ are

$$
\lambda_{1,2}= \pm i \omega_{0}= \pm 1.3587 i, \quad \lambda_{3}=-0.5, \quad \lambda_{4}=-1.3296 \text {. }
$$

And from (7), one has

$$
\begin{gathered}
q=(-0.8311-0.2364 i, 0.5+1.3587 i, 1.26+1.68 i, 1)^{T}, \\
p=(0.0689+0.2062 i,-0.25+0.4503 i \\
0.2987+0.54 i, 0.1192+0.0221 i)^{T},
\end{gathered}
$$




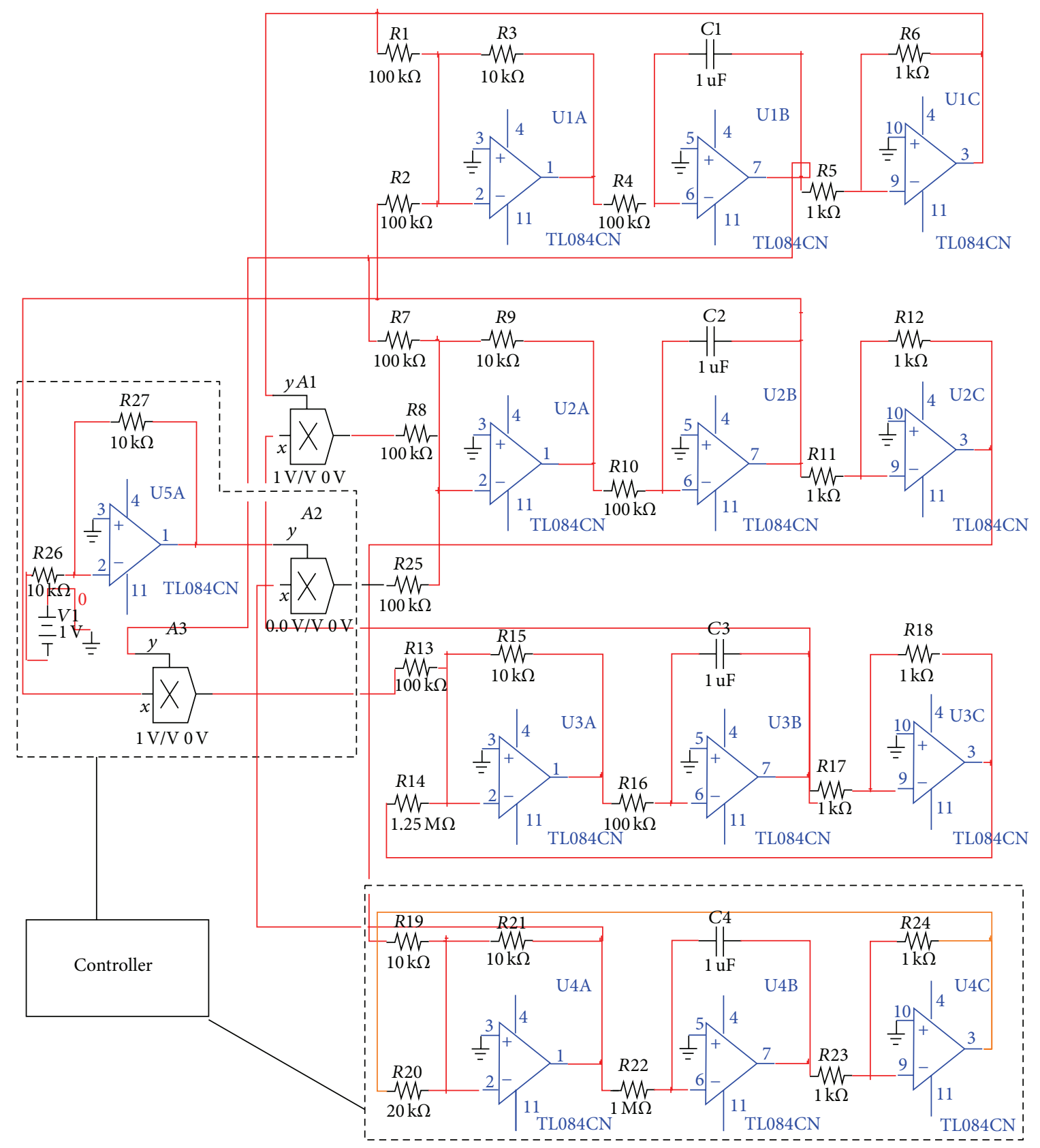

FIGURE 16: Circuit diagram for realizing the chaotic attractor of system (inside the black-dotted box is the controller).

$$
\begin{array}{cc}
B(q, q)=(0,10.6+3.5 i,-0.1628-0.452 i, 0)^{T}, & B\left(\bar{q}, h_{20}\right)=(0,-0.28-0.27 i,-0.779+0.78 i, 0)^{T}, \\
B(q, \bar{q})=(0,1.55,-0.82,0)^{T}, & C(q, q, \bar{q})=\left(0,5.525 k_{2}+5.194 k_{2} i, 0,0\right)^{T}, \\
h_{11}=(0.7975,0.7975,1.55,1.595)^{T}, & H_{21}=\left(0,\left(5.25 k_{2}+0.12\right)+\left(5.194 k_{2}-2.06\right) i,\right. \\
h_{20}=(-0.1567+0.5445 i,-1.627+0.1213 i, & -1.259+2.7 i, 0)^{T}, \\
& G_{21}=-\left(3.721 k_{2}+0.936\right)+\left(1.2 k_{2}+0.699\right) i .
\end{array}
$$$$
0.2712+0.7712 i,-0.0644+0.5907 i)^{T},
$$

Now, consider the family of differential equation (16) $B\left(q, h_{11}\right)=(0,0.2-0.895 i,-0.24+0.96 i, 0)^{T}$, regarded as dependent on the parameter $c$. The real part $\xi$ 


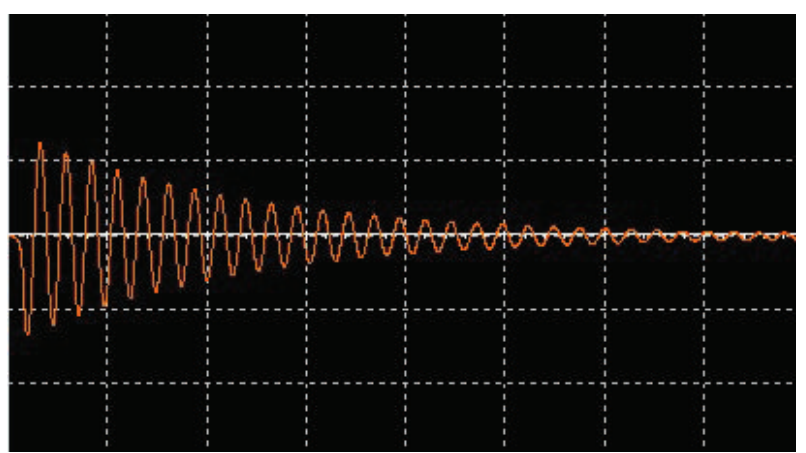

(a) The time history

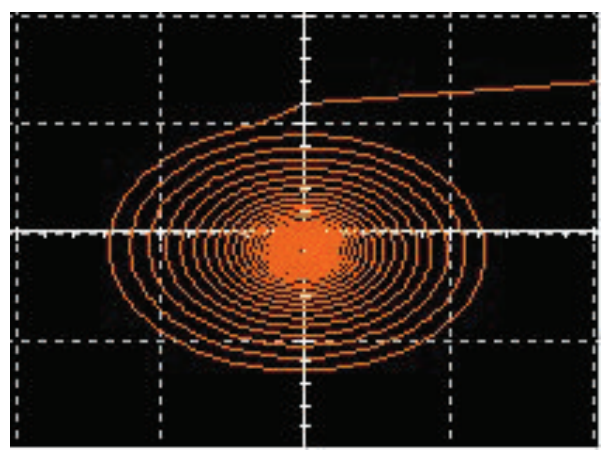

(b) The phase diagram

FIGURE 17: Experimental observations of the time history and phase diagram of system (16) with $a=1, c=0.08, m=0.5, k_{1}=0.8, k_{2}=0.5$, and $b=-0.2$.

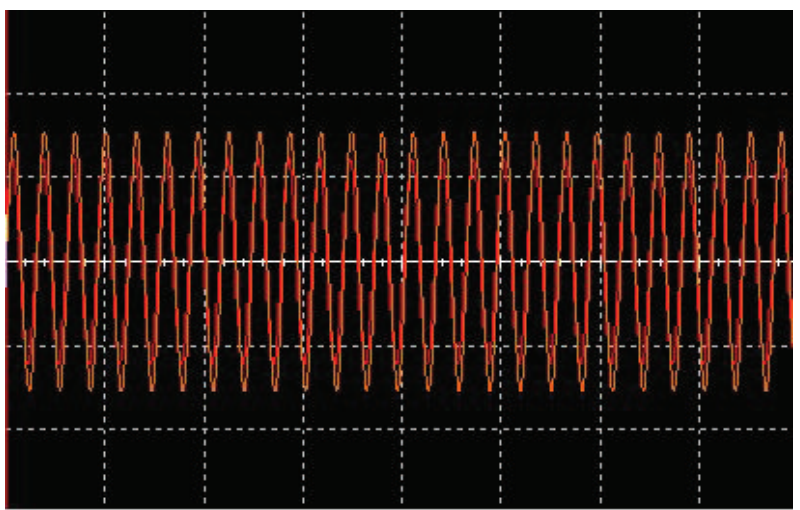

(a) The time history

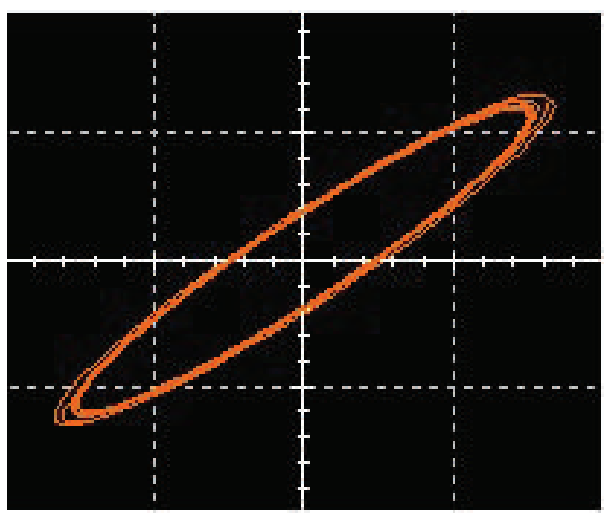

(b) The phase diagram

FIGURE 18: Experimental observations of the time history and phase diagram of system (16) with $a=1, c=0.08, m=0.5, k_{1}=0.8, k_{2}=0.5$, and $b=-0.175$.

of the pair of complex eigenvalues at the critical parameter $c=c_{0}$ verifies

$$
\xi^{\prime}\left(c_{0}\right)=\operatorname{Re}\left\langle p,\left.\frac{d \mathbf{A}}{d b}\right|_{c=c_{0}} q\right\rangle=0.3945>0 .
$$

So, the transversality condition at the Hopf point holds.

Using these calculations, we prove the next theorem.

Theorem 2. Consider the six-parameter family of differential equations (16). The first Lyapunov coefficient associated with the equilibrium $E_{+}$is given by

$$
l_{1}=-1.8605 k_{2}-0.468 \text {. }
$$

If $k_{2} \neq-0.2515$, then $l_{1} \neq 0$ and system (16) has a transversal Hopf point at $E_{+}$for $a=1, b=2$, and $c=0.6998$. More specifically, if $k_{2}>-0.2515$, there exists a stable periodic orbit near the unstable equilibrium point $E_{+}$; if $k_{2}<-0.2515$, there exists a unstable periodic orbit near the asymptotically stable equilibrium point $E_{+}$.

The sign of the first Lyapunov coefficient is determined by $k_{2}$. Observe that the first Lyapunov coefficient vanishes when $k_{2}=-0.2515$. In the following theorem, we study the sign of the second Lyapunov coefficient where the first coefficient vanishes.

Define

$$
\begin{array}{r}
\Omega=\left\{\left(a, b, c, m, k_{1}, k_{2}\right) \mid a=1, b=2, c=0.5,\right. \\
\left.m=0.6998, k_{1}=0.2, k_{2}=-0.2515\right\} .
\end{array}
$$

Considering the system (16) for the parameters $\left(a, b, c, m, k_{1}\right.$, $\left.k_{2}\right) \in \boldsymbol{\Omega}$, one has

$$
\begin{gathered}
C(q, q, \bar{q})=(0,-1.3209-1.3063 i, 0,0)^{T}, \\
H_{21}=(0,-1.2009-3.3663 i,-1.259+2.7 i, 0)^{T}, \\
G_{21}=1.0008 i, \\
h_{30}=(-0.2201+0.1447 i,-0.9726-0.9996 i, \\
-0.0057+0.8692 i,-0.2083+0.167 i)^{T}, \\
h_{21}=(-4.4617+0.4572 i,-4.8018-4.9892 i, \\
-4.6476+2.7212 i,-3.613+2.1395 i)^{T},
\end{gathered}
$$




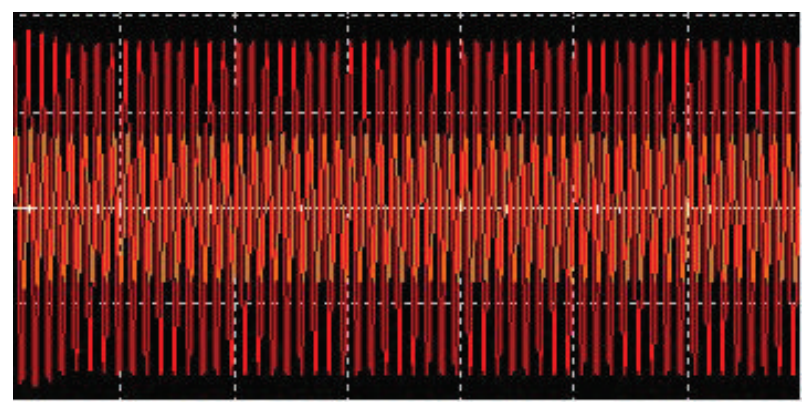

(a) The time history

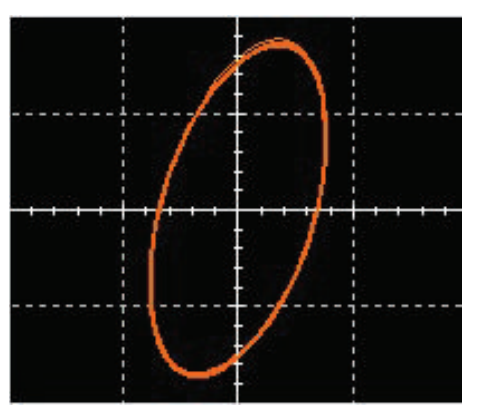

(b) The phase diagram

FIGURE 19: Experimental observations of the time history and phase diagram of system (16) with $a=1, c=0.08, m=0.5, k_{1}=0.8, k_{2}=0.5$, and $b=-0.15$.

$$
\begin{gathered}
h_{31}=(-0.5359-0.1395 i,-1.6454-1.16 i, \\
-1.1993+1.297 i,-0.4584-0.1371 i)^{T}, \\
h_{22}=(2.6732+5.18 i, 2.6732+5.18 i, \\
-9.5872-28.1 i, 5.3464+10.36 i)^{T}, \\
G_{32}=-3.87607-7.1839 i, \\
l_{2}=\frac{1}{12} \operatorname{Re} G_{32}=-0.323005834 .
\end{gathered}
$$

Using these calculations, we prove the next theorem.

Theorem 3. If $\left(a, b, c, m, k_{1}, k_{2}\right) \in \mathbf{\Omega}$, consider the six-parameter family of differential equations (16). The second Lyapunov coefficient associated with the equilibrium $E_{+}$is given by

$$
l_{2}=-0.323005834 \text {. }
$$

As $l_{2} \neq 0$, then system (16) has a transversal Hopf point of codimension 2 at $E_{+}$for parameters in $\boldsymbol{\Omega}$.

Next, we shall give a numerical example of system (16). Let the damping coefficient $m=0.5, k_{1}=0.2$, and $k_{2}=0.5$; the washout filter is designed as

$$
u=0.2(y-0.5 v)+0.5(y-0.5 v)^{3}
$$

The equilibrium $E_{+}$is locally asymptotically stable in the uncontrolled system (1) if we fixed $a=1, b=2$, and $c=$ 0.6998 . Compared with the uncontrolled system (1), the controlled system (16) has a transversal Hopf point at $E_{+}$under this parameter region, as shown in Figures 8 and 9, respectively.

When the parameters $a=1$ and $b=2$ are fixed, while parameter $c$ is varied in the interval $[0,1]$, some different dynamical behaviors of system (1) and system (16) are obtained. The bifurcation diagram of system (1) and system (16) in terms of the parameter $c$ is depicted in Figures 10(a) and 11(a), respectively. As shown in Figure 10(a), while $c$ increases, the system (1) is undergoing the following dynamical routes. When $c \in[0,0.0625)$, there is a perioddoubling bifurcation window. And the system is chaotic when $c \in[0.0625,0.3334)$. Obviously, the uncontrolled system (1) undergoes a Hopf bifurcation at the equilibrium $E_{+}$when $c=$ 0.3334 . When $c \in[0.3334,1)$, the system is period-one orbits. We design a control laws such that our controlled system (16) undergoes a Hopf bifurcation when the parameter $c=0.6998$, as shown in Figure 11. In Figure 11(a), the system is chaotic when $c \in[0,0.575)$. When $c \in[0.575,0.68)$, the system is period-one orbits. The system is chaotic when $c \in$ $[0.68,0.6998)$. And when $c \in[0.6998,1)$, the system is period-one orbits. Figures $10(\mathrm{~b})$ and 11(b) show the corresponding Lyapunov exponent spectrum versus the increasing $b$, respectively. With the analysis performed here one can find that we delayed the Hopf bifurcation.

Letting $a=1, b=2, k_{1}=0.2$, and $k_{2}=0.5$, we can compute the Hopf bifurcation value $c_{0}=0.6998$. The equilibrium is stable when $c=0.8>c_{0}$ and unstable when $c=$ $0.65<c_{0}$, as shown in Figures 12 and 13, respectively. From the formulas in the previous section, we have $l_{1}=-1.39825<$ 0 . Thus, the periodic solution bifurcating from $E_{+}$is supercritical and stable.

\section{Circuit Design for the Chaotic Attractor}

In this section, two possible electronic circuits are given to realize (1) and (16), as shown in Figures 14 and 16, respectively. The first circuit includes three layers, each of which implements one equation of (1). The operational amplifiers and associated circuitry perform the basic operations of addition, subtraction, and integration. The occurrence of the chaotic attractor can be clearly seen from Figures 15(a)-15(c). By comparing them with Figures 1(b)-1(d), it can be concluded that there is a good qualitative agreement between the numerical simulation and the experimental realization. And the second circuit includes four layers, each of which implements one equation of (16). The occurrence of the phase diagram of system (16) can be clearly seen from Figures 17, 18, and 19. By comparing them with Figures 5-7, it can be concluded that there is a good qualitative agreement between the numerical simulation and the experimental realization. 


\section{Concluding Remarks}

In this paper, we consider the problem of anticontrol of Hopf bifurcations; that is, an anticontroller for a Lorenz-like system is designed with desired location and properties by appropriate controls. By the numerical analysis, we prove that Hopf bifurcation occurs when the bifurcation parameter passes through the critical value. In particular, we designed a washout controller such that the equilibrium $E_{0}$ undergoes a controllable Hopf bifurcation, and by adjusting the controller parameters, we delayed Hopf bifurcation phenomenon of the equilibrium $E_{+}$. The proposed anticontrol scheme is effective and easy to manipulate with the aid of symbolic computation. The calculation of the first and second Lyapunov coefficients, which makes the determination of the Lyapunov stability at the equilibriums possible, can make the controlled system exhibit Hopf bifurcation in a much larger parameter region. Besides, we proposed two possible electronic circuits to realize the uncontrolled and the controlled systems. Apparently, there are more interesting problems about this chaotic system in terms of complexity, control, and synchronization, which deserve further investigation.

\section{Acknowledgments}

The authors express their gratitude to the referee for their valuable comments on the first version of the paper. The authors also gratefully acknowledge the support from the National Natural Science Foundation (nos. 11161027 and 61364001), the Key Project of Chinese Ministry of Education (no. 212180), the Fundamental Research Funds for the Universities of Gansu Province (no. 620023), and the Natural Science Foundation of Gansu Province (no. 1010RJZA067), Government of China.

\section{References}

[1] E. N. Lorenz, "Deterministic nonperiodic flow," Journal of the Atmospheric Sciences, vol. 20, pp. 130-141, 1963.

[2] C. Sparrow, The Lorenz Equations: Bifurcations, Chaos, and Strange Attractors, vol. 41, Springer, New York, NY, USA, 1982.

[3] E. Ott, Chaos in Dynamical Systems, Cambridge University Press, Cambridge, Mass, USA, 2nd edition, 2002.

[4] D. S. Chen, H. O. Wang, and G. Chen, "Anti-control of Hopf bifurcations," IEEE Transactions on Circuits and Systems, vol. 48, no. 6, pp. 661-672, 2001.

[5] D. Chen, H. O. Wang, and G. Chen, "Anti-control of Hopf bifurcations through washout filters," in Proceedings of the 37th IEEE Conference on Decision and Control (CDC '98), pp. 3040-3045, December 1998.

[6] Z. Wei and Q. Yang, "Anti-control of Hopf bifurcation in the new chaotic system with two stable node-foci," Applied Mathematics and Computation, vol. 217, no. 1, pp. 422-429, 2010.

[7] Y. C. An, Q. L. Zhang, Y. Zhang, and Z. Su, "Control based on wash-out-filter for Hopf bifurcation of nonlinear systems," Journal of Northeastern University, vol. 29, no. 10, pp. 1381-1384, 2008.

[8] Y.-J. Ma, X.-S. Li, X.-S. Zhou, J. Li, H.-L. Wen, and L.-Y. Jia, "Control of dynamic bifurcation in power system based on
Washout-filter," Power System Protection and Control, vol. 39, no. 23, pp. 54-59, 2011.

[9] J. Sotomayor, L. F. Mello, and D. C. Braga, "Bifurcation analysis of the Watt governor system," Computational \& Applied Mathematics, vol. 26, no. 1, pp. 19-44, 2007.

[10] Y. A. Kuznetsov, Elements of Applied Bifurcation Theory, vol. 112 of Applied Mathematical Sciences, Springer, New York, NY, USA, 3rd edition, 2004.

[11] F. S. Dias, L. F. Mello, and J.-G. Zhang, "Nonlinear analysis in a Lorenz-like system," Nonlinear Analysis. Real World Applications, vol. 11, no. 5, pp. 3491-3500, 2010.

[12] A. Wolf, J. B. Swift, H. L. Swinney, and J. A. Vastano, "Determining Lyapunov exponents from a time series," Physica D, vol. 16, no. 3, pp. 285-317, 1985.

[13] L. F. Mello, M. Messias, and D. C. Braga, "Bifurcation analysis of a new Lorenz-like chaotic system," Chaos, Solitons \& Fractals, vol. 37, no. 4, pp. 1244-1255, 2008. 


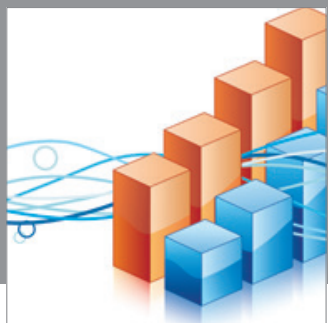

Advances in

Operations Research

mansans

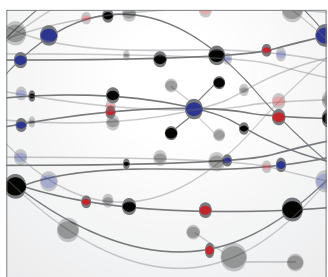

The Scientific World Journal
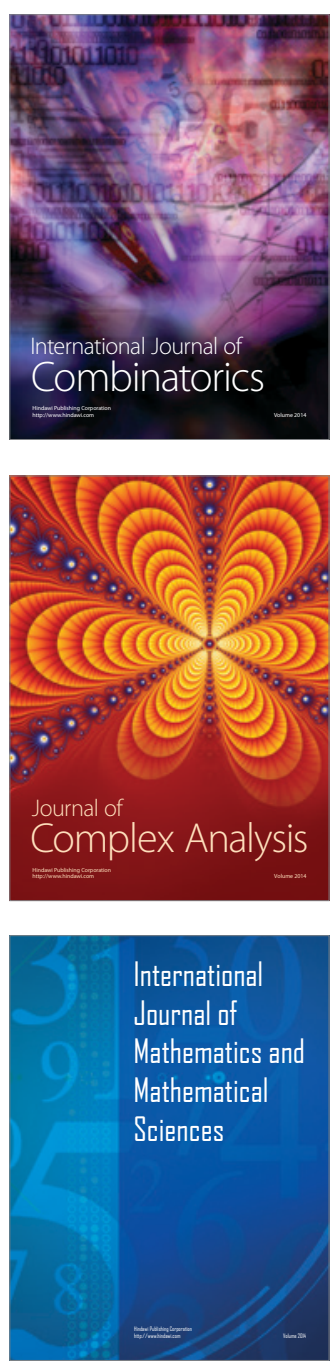
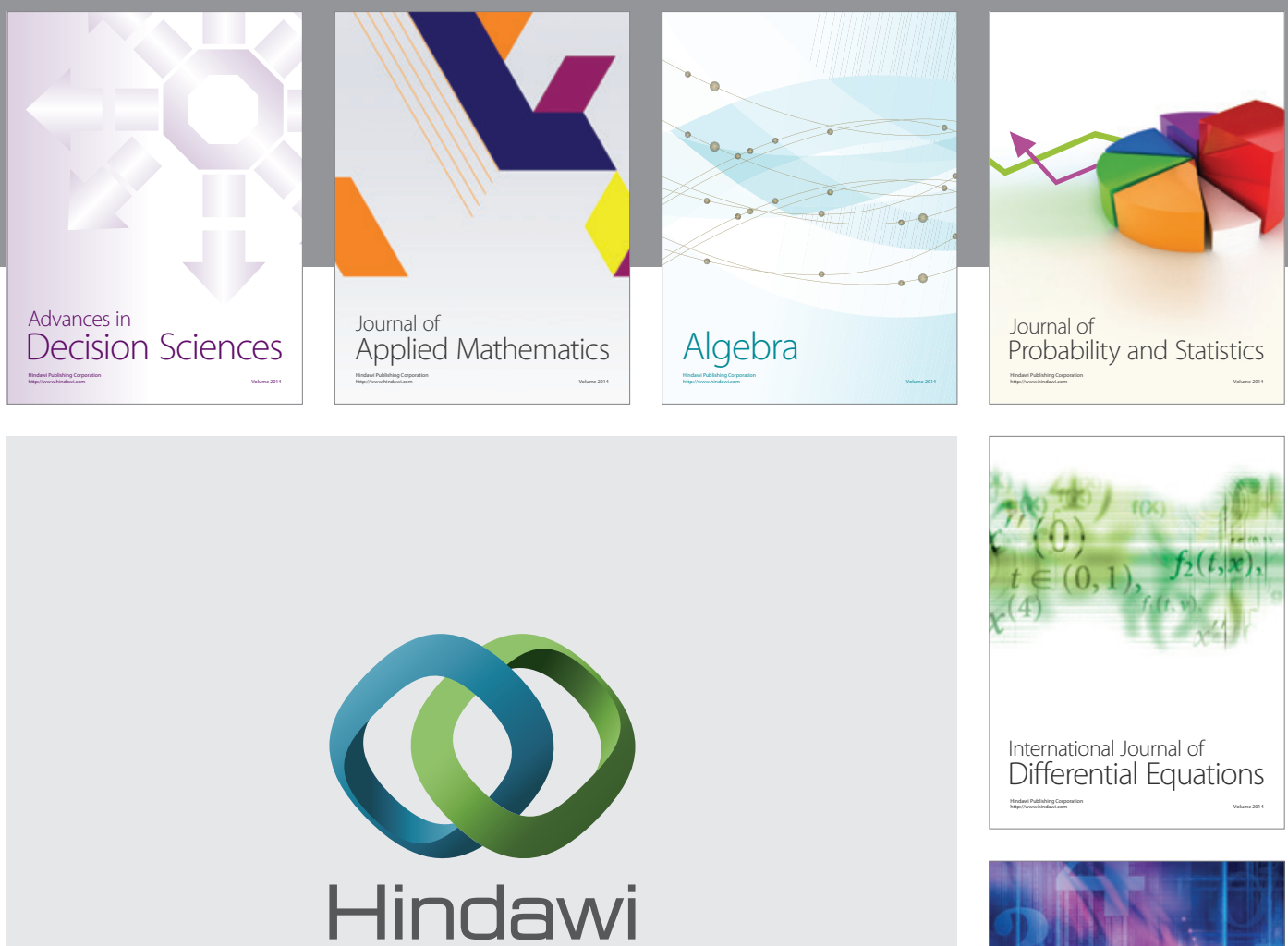

Submit your manuscripts at http://www.hindawi.com
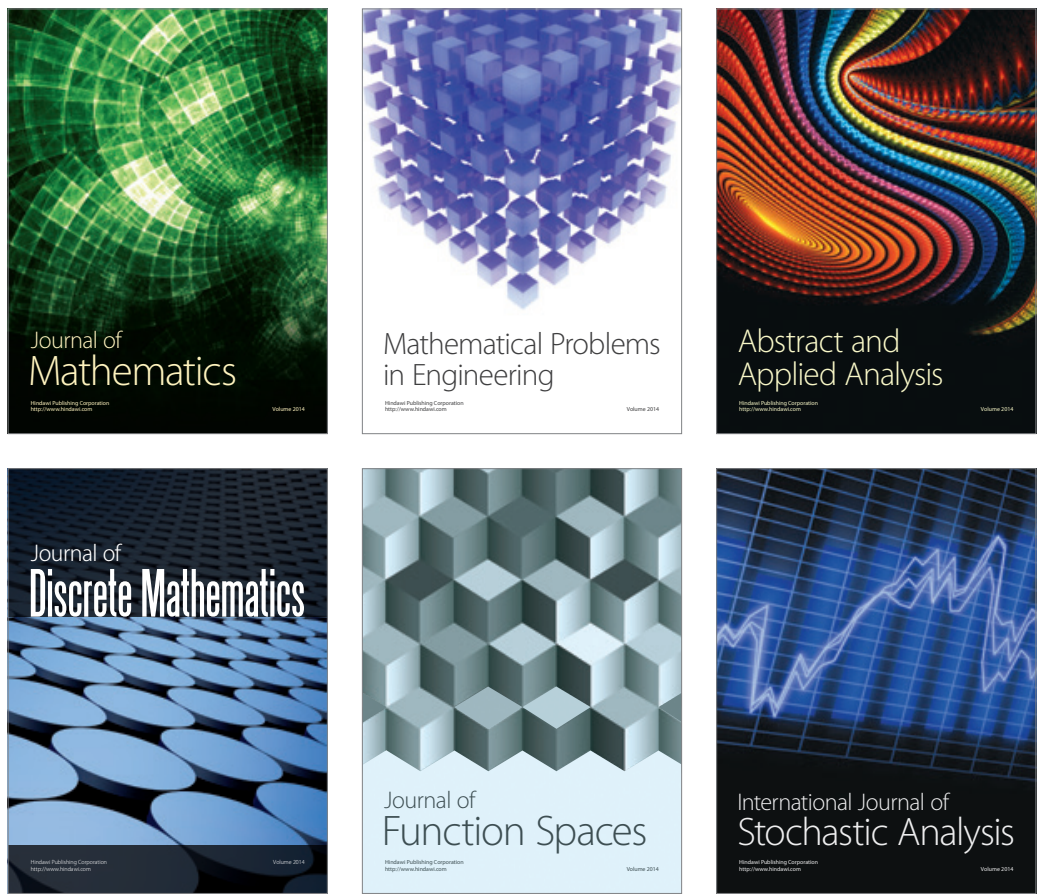

Journal of

Function Spaces

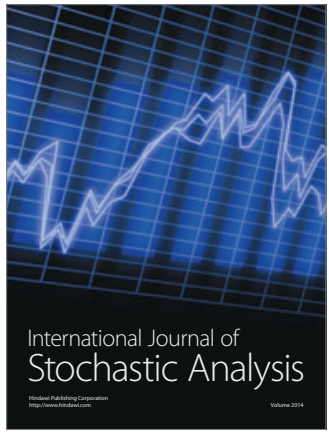

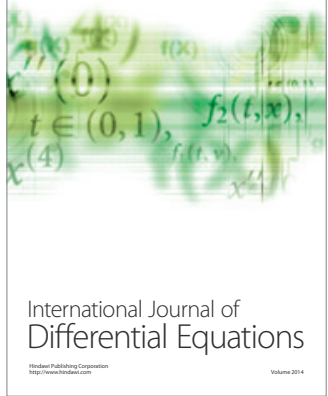
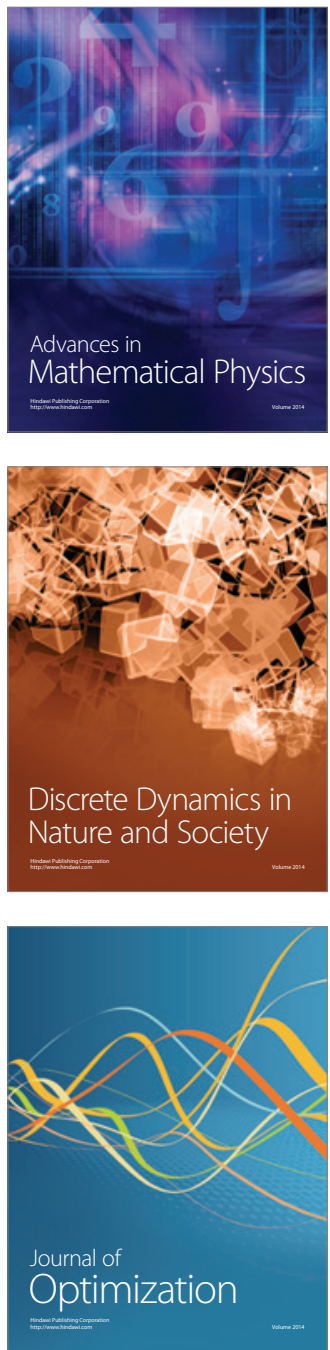